ARTICLE OPEN

\title{
PIAS2-mediated blockade of IFN- $\beta$ signaling: a basis for sporadic Parkinson disease dementia
}

Joana Magalhaes $\mathbb{D}^{1,8}{ }^{1,}$, Emilie Tresse ${ }^{1,8}$, Patrick Ejlerskov ${ }^{1}$, Erling Hu${ }^{1}$, Yawei Liu ${ }^{1}$, Andrea Marin ${ }^{1}$, Alexia Montalant ${ }^{1,2}$, Letizia Satriano ${ }^{1}$, Carsten Friis Rundsten ${ }^{1}$, Eva Maria Meier Carlsen ${ }^{2}$, Rasmus Rydbirk ${ }^{3}$, Ali Sharifi-Zarchi ${ }^{4}$, Jesper Bøje Andersen ${ }^{1}$, Susana Aznar $^{3}$, Tomasz Brudek (iD) ${ }^{3}$, Konstantin Khodosevich (D) ${ }^{1}$, Marco Prinz (iD) ${ }^{5}$, Jean-François Marie Perrier (iD) ${ }^{2}$, Manu Sharma ${ }^{6,7}$, Thomas Gasser ${ }^{7}$ and Shohreh Issazadeh-Navikas (D) ${ }^{1 凶}$

(c) The Author(s) 2021

Familial Parkinson disease (PD) is associated with rare genetic mutations, but the etiology in most patients with sporadic (s)PD is largely unknown, and the basis for its progression to dementia (sPDD) is poorly characterized. We have identified that loss of IFN $\beta$ or IFNAR1, the receptor for IFNa/ $\beta$, causes pathological and behavioral changes resembling PDD, prompting us to hypothesize that dysregulated genes in IFN $\beta$-IFNAR signaling pathway predispose one to SPD. By transcriptomic analysis, we found defective neuronal IFN $\beta$-IFNAR signaling, including particularly elevated PIAS2 associated with sPDD. With meta-analysis of GWASs, we identified sequence variants in IFN $\beta$-IFNAR-related genes in SPD patients. Furthermore, sPDD patients expressed higher levels of PIAS2 mRNA and protein in neurons. To determine its function in brain, we overexpressed PIAS2 under a neuronal promoter, alone or with human a-synuclein, in the brains of mice, which caused motor and cognitive impairments and correlated with intraneuronal phosphorylated ( $p$ )a-synuclein accumulation and dopaminergic neuron loss. Ectopic expression of neuronal PIAS2 blocked mitophagy, increased the accumulation of senescent mitochondrial and oxidative stress, as evidenced by excessive oxDJ1 and $8 \mathrm{OHdG}$, by inactivating ERK1/2-P53 signaling. Conversely, PIAS2 knockdown rescued the clinicopathological manifestations of PDD in Ifn ${ }^{-/-}$mice on restoring mitochondrial homeostasis, oxidative stress, and pERK1/2-pP53 signaling. The regulation of JAK-STAT2PIAS2 signaling was crucial for neurite outgrowth and neuronal survival and excitability and thus might prevent cognitive impairments. Our findings provide insights into the progression of SPD and dementia and have implications for new therapeutic approaches.

Molecular Psychiatry (2021) 26:6083-6099; https://doi.org/10.1038/s41380-021-01207-w

\section{INTRODUCTION}

The etiology of sporadic Parkinson disease (SPD) is unknown, which complicates its treatment. However, studies of rare familial forms of this disorder have prompted its examination genomewide. All PD patients experience motor dysfunction due to loss of dopaminergic neurons in the substantia nigra (SN), correlating with pathogenic a-synuclein (a-syn) aggregates and Lewy bodies [1]. These aggregates amass in an increasingly larger area of the brain as PD progresses and causes such secondary symptoms as dementia [1, 2]. Several mutations have been identified (such as SNCA, LRRK2, DJ1, and Parkin) that are associated with familial PD [3] and some of which have been implicated in SPD [4]. However, familial forms only constitute approximately $5 \%$ of all PD cases $[5,6]$ - the origin of the remaining sporadic cases remains unknown.

Although sPD does not pass through familial inheritance, a genetic predisposition, in combination with environmental factors, could drive its pathology. If this predisposition is linked to several gene variants in the same pathway, the association of each gene with SPD might be weak and thus excluded in large conventional genetic association studies with strict thresholds. To include such variants, one could perform a meta-analysis of genomewide association studies (GWASs) [7] and gene set enrichment analysis (GSEA) [8].

We have reported that the lack of functional interferon-beta (Ifnb) or its receptor, interferon- $\alpha / \beta$ receptor (Ifnar), causes agedependent development of PD-like pathology, including the accumulation of a-syn aggregates and Lewy bodies, in association with motor and cognitive dysfunction [9]. The absence of Ifnar from neuroectodermal cells (Nes ${ }^{\mathrm{cre}} / f_{\text {nar }}{ }^{f / f l}$ ) is sufficient to drive the pathology. Ifnb is a type I IFN and an immunoregulatory cytokine that participates in innate antiviral immune defense and regulates neuroinflammation [10-15]. The progression of PD is associated with neuroinflammation, including the activation of resident

\footnotetext{
${ }^{1}$ Biotech Research and Innovation Centre, University of Copenhagen, Copenhagen, Denmark. ${ }^{2}$ Neuronal Signaling Lab, Faculty of Health and Medical Sciences, University of Copenhagen, Copenhagen, Denmark. ${ }^{3}$ Research Laboratory for Stereology and Neuroscience, Center for Translational Research, Bispebjerg-Frederiksberg Hospital, Copenhagen, Denmark. ${ }^{4}$ Department of Stem Cells and Developmental Biology, Cell Science Research Center, Royan Institute for Stem Cell Biology and Technology, ACECR, Tehran, Iran. ${ }^{5}$ Institute of Neuropathology, Signalling Research Centres BIOSS and CIBSS, Center for Basics in NeuroModulation (NeuroModulBasics), Faculty of Medicine, University of Freiburg, Freiburg, Germany. ${ }^{6}$ Hertie Institute for Clinical Brain Research, University of Tübingen, Tübingen, Germany. ${ }^{7}$ Centre for Genetic Epidemiology, Institute for Clinical Epidemiology

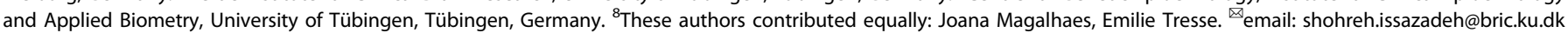


6084

microglia and astrocytes, which exacerbates the pathology in the late stage of the disease [16]. However, the function of Ifnb signaling in neurodegeneration and neuroinflammation in SPD patients has not been determined.

The transcription and subsequent secretion of type I IFNs are induced on viral infection via Toll-like receptor signaling [11], MAVS [17], and RIG-I [18] and is orchestrated by STING-CGAS [19]. Extracellular IFN- $\beta$ activates the JAK-STAT pathway on binding its receptor, IFNAR, inducing intracellular phosphorylation and nuclear translocation of STAT proteins for transcriptional initiation through interferon-stimulated responsive elements and gammaactivated sequences [20]. STATs are negatively regulated by protein inhibitors of STAT (PIASs), but their involvement in PD is unknown.

Protein inhibitor of STAT2 (PIAS2) is a negative regulator of STAT [21] and IFN- $\beta$ signaling [22] and has SUMO E3 ligase activity [23]. PIAS2 was recently shown to stabilize $\alpha$-syn by counteracting its ubiquitin-mediated degradation, promoting its intracellular aggregation [24].

In light of our recent findings of the relevance of defective IFN $\beta$ -IFNAR1 signaling to cause a Parkinson disease dementia (PDD)like pathology in mice [9], we examined whether and how defective type I IFN signaling is associated with sporadic PD and its progression to dementia. Notably, genes in this pathway were significantly dysregulated in SPD patients-especially those with dementia. Moreover, we identified single-nucleotide polymorphism (SNP) variants in several of these genes that correlated with SPD, including PIAS2. PIAS2 was upregulated in the neurons of SPD patients, particularly in the brains of patients with SPDD. These findings prompted us to study how PIAS2 impacts the clinical and pathological aspects of PD-specifically, the cognitive functions that are associated with its progression to dementia. Overexpression of PIAS2 alone was sufficient to cause PD-like dementia and major pathological trademarks of PDD in mice, whereas its knockdown in vivo reversed the PD-like dementia and pathology in Ifn $b^{-1-}$ mice, implicating it as a driver of PD pathology.

\section{MATERIALS AND METHODS}

Brain samples from sporadic PD patients and healthy controls Human brain samples for reverse-transcription quantitative real-time (RT-q) PCR and IHC were dissected from the medial frontal gyrus, snap-frozen, and stored at $-80^{\circ} \mathrm{C}$ prior to analysis. Sporadic PD brain samplessporadic Parkinson disease nondementia (sPDND) $(n=10)$, sPDD $(n=3)$, and healthy controls (HCS) $(n=10)$-were acquired from the Harvard Brain Tissue Resource Center (Harvard Medical School, USA), Bispebjerg Hospital Brain Bank (Copenhagen, Denmark/BBH-2017-001, I-suite nr: 05190), and Netherlands Brain Bank (the Netherlands) [ethics (DNK) approval, jr. no. $\mathrm{H}-$ 16025210]. All diagnoses were confirmed by postmortem pathology.

\footnotetext{
Affymetrix microarray analysis

Publicly available microarray data from PDD $(n=13)$, PDND $(n=15)$, and HCs $(n=14)$ were obtained from an earlier study [PMID:18649390] [25]. The preprocessed and normalized data were acquired from the Affymetrix Human Genome U133 Plus 2.0 Array. To ensure consistency, the public data were renormalized in $\mathrm{R}$ using the normalize quantile function in preprocessCore. Three other independent datasets (GSE7621, GSE20141, GSE49036) on SN tissue from the postmortem brains of HCs $(n=25)$ and PD patients $(n=45)$ were pooled to validate the data from [25]. Samples were run on the Affymetrix Human Genome U133 Plus 2.0 Array and processed in $\mathrm{R}$ using the ReadAffy parser in affy. Preprocessing and normalization were performed with the frma package using the "robust weighted average" option for probe summarization.

Microarrays of Ifnb $b^{+/+}$and Ifnb $b^{-/-}$CGN cultures with or without 24-h rIFN- $\beta$ treatment $(100 \mathrm{U} / \mathrm{ml})$ were set up in triplicate, and the extracted cDNA was applied to the Affymetrix Mouse Genome 4302.0 microarray chip (SCIBLU, Affymetrix). GEO accession number GSE63815. Data were analyzed with Arraystar 3 (DNA STAR Inc.) and quantile-normalized and processed using the RMA (Affymetrix) algorithm. Data were preprocessed in $\mathrm{R}$ using the affy package and RMA function.
}

\section{Class prediction, gene set enrichment analysis, and interactive} pathway map

BRB ArrayTools v4.5.0 (Biomedical Research Branch, NIH), using seven algorithms (1-nearest neighbor, 3-nearest neighbors, compound covariate predictor, diagonal linear discriminant analysis, nearest centroid, support vector machines, and Bayesian compound covariate classifier), was used to identify 638 differentially expressed genes $(p<0.01)$ between PDD and PDND. Leave-one-out crossvalidation, based on 1000 random permutations, was used to compute the misclassification rate. Functional annotation and pathway analysis were performed by GSEA (v2.2.2), and heatmaps were generated by extracting the core enriched genes (Molecular Signatures Database v5.1, Broad Institute). An interactive pathway map that compared gene expression in the type I IFN pathway, obtained from WikiPathways [26], in PD versus HC was created using PathVisio v3.2.1 [27]. This pathway is based on 32 publications, listed in the bibliography of the pathway. The log2-scale fold-change values were shown on a gradient from -1 to +1 .

\section{Meta-analysis of SNPs from IPDGC}

We leveraged summary statistics data from the International Parkinson Disease Genomics Consortium (IPDGC), which are described elsewhere [4]. In brief, we selected genes that were directly downstream of type I interferon receptor and performed a meta-analysis, comparing overlapping SNPs with two datasets by the IPDGC: 7,689,524 SNPs from 5333 PD cases and 12,019 HCs [4] and 7,893,274 SNPs from 13,708 PD cases and 95,282 HCs [28]. We examined the $100 \mathrm{~kb}$ upstream and downstream to encompass the signaling pathway and used fixed-effects meta-analysis as the primary method of analysis (Table 1 and Data File 1).

\section{Mice}

Ifn $\mathrm{f}^{-/-}$mice were backcrossed for 20 generations to B10.RIII [9] or C57BL6 mice. The Ifnar $1^{-/}$and nes ${ }^{\text {Cre }}:$ Ifnarr $1^{f / f l}$ mice were on the C57BL6 background [13]. The wild-type (WT) animals were $\operatorname{lfn} b^{+/-}$, Ifn $b^{+/+}$, or Ifnar $^{+/+}$littermates. Mice were housed in standard facilities. Sex- (equal proportion male and female), age- (8-9-week old at injection), and weightmatched $(28-31 \mathrm{~g})$ mice were used in the experiments according to the ethical committees in Denmark and our institutional review boards (201315-2934-00807, 2018-15-0201-01572).

\section{AAV6 production}

HEK293 cells (80\% confluent) were transfected with pDP6 AAV6 [29] and pAAV-SYN1-mCherry-mouse PIAS2, pAAV-SYN1-mCherry-mouse mutant PIAS2 ${ }_{\text {C362A, }}$ or PAAV-SYN1-mCherry (Vector Builder) by $\mathrm{CaCl} 2$ method. After $72 \mathrm{~h}$, cells were harvested by scraping, centrifuged at $200 \mathrm{~g}$, and lysed with $5 \mathrm{ml}$ Tris $0.05 \mathrm{M}, \mathrm{NaCl} 0.15 \mathrm{M}, \mathrm{MgCl}_{2} 0.005 \mathrm{M}$. Three freeze-thaw cycles were then performed. Benzonase (Invitrogen) was added to $50 \mathrm{U} / \mathrm{ml}$ and incubated for $30 \mathrm{~min}$ at $37^{\circ} \mathrm{C}$, and the mixture was centrifuged at $4500 \mathrm{~g}$ for $15 \mathrm{~min}$. Supernatant was collected, and $5 \mathrm{ml}$ lysis buffer was added to the cell pellet. After centrifugation at $4500 \mathrm{~g}$ for $15 \mathrm{~min}$, the second supernatant was collected. Both supernatants were pooled and passed through 0.45 - and $0.22-\mu \mathrm{m}$ Acrodisc filters. The filtered supernatant was added to an iodixanol gradient $(1.5 \mathrm{ml}$ for each step from 0 to 15 to 25 to 40 and $60 \%)$ and centrifuged at $50,000 \mathrm{rpm}$ for $2 \mathrm{~h}$ at $4{ }^{\circ} \mathrm{C}(70.1 \mathrm{Ti}$ Rotor Beckman). The adeno-associated virus (AAV) fraction was collected between the fractions at 60 and $40 \%$, concentrated, and bufferexchanged to PBS by centrifugation at $2000 \mathrm{~g}$ at $4{ }^{\circ} \mathrm{C}$ using an Amicon $100 \mathrm{~K}$ filter. Finally, the AAV was passed through a 0.22 Acrodisc filter. Titration was performed by RT-qPCR for WPRE using fw $5^{\prime}$-CCGTTGTCAGGCAACGTG-3' and rev 5'-AGCTGACAGGTGGTGGCAAT-3' as primers. The titers that we obtained were $3.5 \mathrm{e} 10^{6} / \mu \mathrm{l}$ for AAV mCherry, $9.0 \mathrm{e} 10^{6} / \mu \mathrm{l}$ for mutPIAS2, $7.4 \mathrm{e} 10^{6} / \mu \mathrm{l}$ for WT-PIAS2, and $3.0 \mathrm{e} 10^{6} / \mu \mathrm{l}$ for hSNCA.

\section{Stereotactic injection of AAV6 PIAS2 and PIAS2 siRNA}

Two-month-old WT, Ifnar $1^{-1-}$, and $1 \mathrm{fnb}^{-/-}$C57BL6 mice underwent stereotactic brain injections of AAV6 PIAS2-mCherry (pAAV\{Exp\}-SYN1>mCherry(ns):mPias2\{NM_008602.4\}:WPRE\}; Vector Builder) or AAV6 PIAS2 $2362 A$-mCherry (pAAV\{Exp\}-SYN1>mCherry(ns):mPias2\{NM_008602.4\} *(C362A):WPRE\}; Vector Builder) - with or without AAV6 human SNCA (pAAV\{Exp\}-SYN1>hSNCA\{NM_000345.5\}:WPRE\}; Vector Builder). For the siRNA knockdown experiments, Accell siRNA against PIAS2 and a scrambled control were obtained from Dharmacon. AAV6 vectors were prepared as described. Isoflurane was used for general anesthesia through 
Table 1. Putative pathogenic rare risk variants in PIAS2, JAK2, TYK2, and AKT1 associated with Parkinson disease.

\begin{tabular}{|c|c|c|c|c|}
\hline PIAS2/chr18 & Allele1 & Allele2 & SE & Meta_P \\
\hline chr18:42747317 & $\mathrm{t}$ & c & 0.1619 & 0.04771 \\
\hline chr18:42719495 & $\mathrm{a}$ & $\mathrm{g}$ & 0.1628 & 0.04853 \\
\hline JAK2/chr9 & Allele1 & Allele2 & SE & Meta $P$ \\
\hline chr9:4977057 & $\mathrm{t}$ & c & 0.1056 & 0.02126 \\
\hline rs7863708 & $\mathrm{t}$ & c & 0.0637 & 0.02209 \\
\hline rs7026646 & a & g & 0.0618 & 0.02856 \\
\hline rs7857730 & $\mathrm{t}$ & g & 0.0256 & 0.03138 \\
\hline rs2031906 & $\mathrm{t}$ & c & 0.0256 & 0.03521 \\
\hline rs10117591 & a & g & 0.0254 & 0.03582 \\
\hline rs7847294 & a & c & 0.0254 & 0.03743 \\
\hline rs10115312 & $\mathrm{t}$ & g & 0.0262 & 0.03766 \\
\hline rs7861599 & $\mathrm{t}$ & c & 0.0257 & 0.03947 \\
\hline rs4372063 & a & g & 0.0262 & 0.04055 \\
\hline rs2149556 & $\mathrm{t}$ & c & 0.0256 & 0.04097 \\
\hline rs7034878 & a & c & 0.0256 & 0.04097 \\
\hline rs9987451 & $\mathrm{t}$ & c & 0.0254 & 0.04242 \\
\hline rs10815158 & a & g & 0.0254 & 0.04322 \\
\hline rs3780378 & $\mathrm{t}$ & c & 0.0254 & 0.04322 \\
\hline rs10815146 & a & $\mathrm{t}$ & 0.0254 & 0.0441 \\
\hline rs10815144 & a & g & 0.0254 & 0.04417 \\
\hline rs7852988 & a & c & 0.0254 & 0.04417 \\
\hline rs7034753 & a & g & 0.0254 & 0.04491 \\
\hline rs4587378 & $\mathrm{t}$ & c & 0.0261 & 0.04607 \\
\hline rs1328918 & a & g & 0.0256 & 0.04644 \\
\hline rs4282620 & a & c & 0.0256 & 0.04671 \\
\hline rs7019858 & $\mathrm{t}$ & c & 0.0256 & 0.04671 \\
\hline rs7033052 & c & $\mathrm{g}$ & 0.0258 & 0.04939 \\
\hline TYK2/chr19 & Allele1 & Allele2 & SE & Meta $P$ \\
\hline chr19:10349680 & a & g & 0.0713 & 0.007884 \\
\hline chr19:10349007 & a & g & 0.1136 & 0.04181 \\
\hline AKT1/chr14 & Allele1 & Allele2 & SE & Meta $P$ \\
\hline rs3803304 & c & g & 0.0344 & 0.001726 \\
\hline chr14:104314779 & a & g & 0.0443 & 0.004011 \\
\hline chr14:104314770 & a & g & 0.0526 & 0.008963 \\
\hline rs10149779 & a & g & 0.0475 & 0.01566 \\
\hline rs3730358 & a & g & 0.0621 & 0.02332 \\
\hline chr14:104328847 & a & $\mathrm{g}$ & 0.0631 & 0.04163 \\
\hline rs2498800 & $\mathrm{t}$ & c & 0.0284 & 0.04245 \\
\hline rs2494734 & c & g & 0.029 & 0.04262 \\
\hline rs12590657 & c & g & 0.0293 & 0.04303 \\
\hline rs2498797 & $\mathrm{t}$ & c & 0.029 & 0.04405 \\
\hline rs3001371 & $\mathrm{t}$ & c & 0.0292 & 0.04445 \\
\hline rs2494733 & c & g & 0.0288 & 0.04454 \\
\hline rs2494731 & c & g & 0.0281 & 0.04508 \\
\hline chr14:104328399 & a & g & 0.063 & 0.04511 \\
\hline rs1130233 & $\mathrm{t}$ & c & 0.0298 & 0.04594 \\
\hline
\end{tabular}

inhalation. Before injection $4 \%$ lidokain ointment is applied topically before a skin incision (max $0.5 \mathrm{~cm}$ ).

Mice were placed in a stereotactic frame, and AAV or siRNA was injected using a 10- $\mu$ l Hamilton syringe. Two microliters of solution was injected unilaterally or bilaterally into the $\mathrm{SN}$, striatum, and prefrontal cortex 16 days or 1 month later, their behavior was tested, and their brains were dissected. The AAV solution was infused at $2 \mu \mathrm{l} / \mathrm{min}$, and the needle was left in place for an additional $45 \mathrm{~s}$ before being slowly retracted. Injections were performed at the following coordinates: $\mathrm{SN}$ : antero-posterior: $-3 \mathrm{~mm}$, medio-lateral: $-/+1.3 \mathrm{~mm}$, dorso-ventral: $-3.9 \mathrm{~mm}$; striatum: antero-posterior: $+0.3 \mathrm{~mm}$, medio-lateral: $-/+2.3 \mathrm{~mm}$, dorso-ventral: $-2.9 \mathrm{~mm}$; prefrontal cortex: antero-posterior: $+1.8 \mathrm{~mm}$, medio-lateral: $-/+1 \mathrm{~mm}$, and dorsoventral: $-2.5 \mathrm{~mm}$-relative to the bregma per a stereotactic atlas.

After the injections, the mice were monitored daily and if signs of reduced well-being, such as inactivity, reduced grooming, loss of appetite and weight (more than 20\%) were observed, the mice were euthanized. Typically the mice did not show any sign of inactivity or weigh loss.

\section{Behavioral measurements}

Groups were sex and weight matched. The experimenter was blind to the genotype and conditions. All animal groups for each model were tested in a same battery. Fifteen and 30 days post injections were selected for behavioral analysis as cognitive impairments might take longer to exhibit while motor dyscoordinations are often appears earlier. Cognition assessments were privileged to be tested prior to motor tests to avoid interfering with behaviors.

An accelerating RotaRod (TSE Systems $\mathrm{GmbH}$ ) was used to assess motor coordination as previously described [9]. After a pretraining period at 5 rpm for $1 \mathrm{~min}$, the speed was increased from 5 to $40 \mathrm{rpm}$ over $3 \mathrm{~min}$ with increment of $1 \mathrm{rpm}$ every $5 \mathrm{~s}$ and kept at $40 \mathrm{rpm}$ for another $2 \mathrm{~min}$. Time before falling was recorded, with a maximum duration of $5 \mathrm{~min}$ for each trial. Four trials were performed with at least $10 \mathrm{~min}$ of rest between trials.

Cylinder test was conducted on asymmetrical forelimb use during vertical exploration to measure akinesia in mice. Climbing test was used to determine motor function by measuring their ability to stand and climb. One 5-min trial was performed for each test.

Gait test was administered to measure motor performance and loss of coordination. The fore and hind paws were painted with different dyes to distinguish footprints. Then, the mice were allowed to walk through a corridor to a safe box. The mice were trained three times on the day before the trial. On the day of the trial, various aspects of the footprints in the corridor were measured: total distance from the first to last step with each paw, stride length, and degree of overlap.

To measure cognitive impairment, the Barnes maze was applied. The Barnes maze consists of a circular platform with 20 circular holes around its circumference, under one of which lay an escape box, oriented by visual clues in front of the platform. On day 1 (day 15 post injection), the mice were subjected to one habituation trial $(30 \mathrm{~s}$ in the center of the maze and 2 min inside the escape box) and three training trials of $180 \mathrm{~s}$ each (or until escape box was reached). The next day, to test spatial short-term memory, the test was administered twice (probe, $90 \mathrm{~s}$ ) but with the box removed. Finally, at day 15 (day 30 after injection), the mice were subjected to a new round twice (probe, $90 \mathrm{~s}$ ) to test long-term memory. An automated video tracking system, Ethovision 3.1 (Noldus Information Technology), recorded the time that elapsed to find the escape hole during the learning trials and that in the area of the escape hole during the probe tests. Zone 1 corresponds to the quarter part of the maze including goal box.

\section{Cell cultures}

Cerebellar tissue was dissected from 7-day-old mice, and in vitro cultures were established as described [30].

Cortical neuron (CN) cultures were obtained from the cortex of 1-day-old mice, as described (Brewer and Torricelli [31]). Neurons were cultured on poly-D-lysine-coated plates in neurobasal medium (Gibco) that contained B27 (2\%) and gentamicin.

SHSY5Y (Lundbeck) stock cells were kept in DMEM with GlutaMax and $10 \%$ fetal bovine serum (FBS) and plated on laminin-coated plates. Neuronal differentiation was induced with retinoic acid $(1 \mu \mathrm{m})$ in DMEM with GlutaMax and 2\% FBS for 4 days. Recombinant IFN- $\beta$ (R\&D Systems, UK) was added to neuronal cultures at $30 \mathrm{U} / \mathrm{ml}$ for 3 days or $100 \mathrm{U} / \mathrm{ml}$ for $24 \mathrm{~h}$.

\section{siRNA transfection of cell cultures in vitro}

siRNA-mediated knockdown was performed using the Accell SMART pool system from Dharmacon (Thermo Scientific) or Sigma, combining 3-4 siRNAs per transduction using $10 \mathrm{nM}$ of each sequence. SHSY5Y cells and CNs were transfected with siRNAs in Accell or Lipofectamine 2000 
6086

(Invitrogen) per the manufacturer, incubated for $48 \mathrm{~h}$, and treated with IFN$\beta, A Z D$, or control for another $24 \mathrm{~h}$ before analysis.

Delivery efficiency and siRNA specificity were examined by WB. The Accell nontargeting control and universal negative control 1 (UNC1; Sigma) were the siRNA controls [32].

The siRNA sequences were as follows: STAT1\#1: 5' CUG UGA AGU UGA GAC UGU U 3', STAT1\#2: 5' CUC AUU CCG UGG ACG AGG U 3', STAT1\#3: 5' CCU GAU UAA UGA UGA ACU A and STAT1\#4: $5^{\prime}$ CGU AAU CUU CAG GAU AAU U 3'. STAT2\#1: SASI_Hs01_00111824; STAT2\#2: SASI_Hs01_00111825; STAT3\#3: HA03445053. The siRNAs were a mixture of $10 \mathrm{nM}$ of each sequence.

The target sequence of the PIAS2 Mission ${ }^{\circledR}$ siRNA (cat. no. EHU122581) was: AGA AAA AGC CCA CCT GGA TाT GTC CTG TGT GTG ACA AAA AAG CTG CCT ATG AAA GTC TAA TAT TAG ATG GGC TाT TTA TGG AAA TTC TCA ATG ACT GTT CTG ATG TAG ATG AGA TCA AAT TCC AAG AAG ATG GT CTT GGT GTC CAA TGA GAC CGA AGA AAG AAG CTA TGA AAG TAT CCA GCC AAC CGT GTA CAA AAA TAG AAA GTT CAA GCG TCC TCA GTA AGC CTा GTT CAG TGA CTG TAG CCA GTG AGG CAA GCA AGA AGA AAG TAG ATG TTA TTG ATC TTA CAA TAG AAA GCT CTT CTG ACG AAG AGG AAG ACC CTC CTG CCA AAA GGA AAT GCA TCT TTA TGT CAG AAA CAC AAA GCA GCC CAA CCA AAG GGG TTC TCA TGT ATC AGC CAT CTT CTG TAA GGG TGC CCA GTG TGA CTT CGG TTG ATC CTG CTG CTA TTC CGC CTT CAT TAA CAG ACT ACT CAG TAC CAT TCC ACC ATA CGC CAA TAT CA.

\section{Immunohistochemistry, immunofluorescence, and transmission electron microscopy}

For $\mathrm{IHC}$ and $\mathrm{IF}$, mice were perfused, and their brains were fixed in $4 \%$ paraformaldehyde (PFA) and paraffin-embedded, or their brains were dissected and snap-frozen before sectioning. In vitro neuronal cultures were fixed in 4\% PFA before staining. Tissues and cells were stained as described [30].

IF images were taken with a Zeiss LSM510 confocal scanning microscope and an IN Cell Analyzer 2200 automated microscope. IHC images were taken with a NanoZoomer 2.0-HT digital slide scanner or Olympus BX51 microscope. Images were processed and quantified in ImageJ (Fiji version), IN Cell Investigator, CellProfiler, Zeiss Zen, and Adobe Photoshop.

Polyclonal and monoclonal rabbit anti- $\beta$-amyloid ${ }_{1-42}$ (1:50, Millipore, AB5078P; and Invitrogen, clone: H31L2, 700254, respectively), mouse anti- $\beta$-amyloid $_{1-16}(1: 500$, clone 6E10, 803015, Biolegend, US), and biotin-labeled secondary antibodies (Vector Laboratories) were used for TEM, after which the samples were treated with $0.3 \% \mathrm{H}_{2} \mathrm{O}_{2}$. IF staining was performed with DAPI (DAKO), rabbit anti-phosphorylated (Ser129) a-synuclein (1:300, AbCam, clone EP1646Y, Ab51252), rabbit anti-PIAS2 (1:100, Neo Scientific, A5654), mouse anti- $\beta$ III-tubulin (1:50, Santa Cruz, clone TUJ-1, Sc-58888), and Congo Red solution (0.2\%, SigmaAldrich, US) and Alexa Fluor -488 and -568 , streptavidin-Alexa Fluor -488 and -568 (Invitrogen), biotinylated rabbit anti-sheep (Vector Laboratories), and streptavidin-CY3 (1:200, US Biological) as secondary antibodies.

\section{Antibodies and reagents}

The primary antibodies were tyrosine hydroxylase (TH) (Millipore, WB 1:2000 IHC 1:200, abcam IF/IHC 1:25), alpha-synuclein (Cell Signaling, WB 1:3000), phospho alpha-synuclein (S129) (Abcam WB 1:1000, IF/IHC 1:100), human alpha-synuclein (Thermo Scientific IHC 1:100), PIAS2 (Neoscience, WB 1:3000, IF 1:50), rabbit anti-PIAS2 (1:100, Neo Scientific, A5654), vinculin (Sigma, WB 1:100000), ERK1/2 (Cell Signaling, WB 1:1000), phospho-ERK1/2 (Cell Signaling, WB 1:1000, IF 1:100), P53 (Abcam, WB 1:500, IF 1:100), phospho-P53 (Abcam, WB 1:500, IF 1:100), DJ1 (Abcam, WB 1:5000), OxDJ1 (Cys106) (Abcam, WB 1:10000, IF 1:100), DUPS1 (Abcam, WB 1:1000), sumo1 (Millipore, WB 1:500), 8OHdG (Santa Cruz Technologies, IF/IHC 1:50), $\beta$ tubulin III (Abcam, IF 1:50), mouse anti- $\beta$ III-tubulin (1:50, Santa Cruz, clone TUJ-1, sc-58888), TOM20 (Santa Cruz Technologies, IF 1:50; Abcam, IF 1:100), Coxl (Abcam, IF 1:140), Optineurin (Santa Cruz, WB 1:500, IF 1:100), and Hsp60 (Santa Cruz Technologies, IF 1:50).

The secondary antibodies were biotinylated anti-rabbit (Vector Laboratories); anti-mouse, -rabbit, -goat, and -chicken coupled to Alexa Fluor-488, -568 , and -633 (1:1000, Invitrogen); streptavidin-Alexa Fluor -488 and -568 (Invitrogen); biotinylated rabbit anti-sheep (Vector Laboratories); and streptavidin-CY3 (1:200, US Biological). Nuclei were stained with DAPI (1:30.000, DAKO). Nissl (ThermoFisher Scientific, 1:200) was used to stain neurons.

\section{Quantitative real-time PCR}

Total RNA was isolated using the QIAGEN RNeasy microkit (QIAGEN), reverse-transcribed, amplified, and quantified using SYBR Green (Bio-Rad). RT-qPCR reactions were run with LightCycler 480 software (Roche). Relative mRNA expression was normalized to Gapdh.

All murine primers were purchase from QIAGEN. The human primers are listed below. The DNA oligomers were designed and ordered from TAG Copenhagen A/S, except for the all-in-One ${ }^{\mathrm{TM}} \mathrm{RT}-\mathrm{qPCR}$ primers for Ifna14, Ifng, and Tnf, which were purchased from GeneCopoeia.

\section{RT-qPCR primers}

\begin{tabular}{|c|c|c|}
\hline Gene & Forward primer & Reverse primer \\
\hline STAT2 & ATTCTGCAGCATTTCCCACT & GCTCATACTAGGGACGGGAAG \\
\hline PIAS2 & TGTTGAGGTGTCAAAGCAAAA & TGATGTTCTCATCAAGCCCA \\
\hline IRF3 & TAAACGCAACCCTTCTITGC & GATGCACAGCAGGAGGATTT \\
\hline IRF9 & CATGGCTCTCTTCCCAGAAA & AGCTCTTCAGAACCGCCTAC \\
\hline IFNB1 & TGGAGAAGCACAACAGGAGA & AACCTITCGAAGCCTITGCT \\
\hline IFNA14 & TTGATTCAACTTGTGGTGGTT & TGGTTCATCATGGAAATGATTC \\
\hline IFNA17 & АTTCTTCCCATTTGTGCCAG & ATGGCCCTGTCCTITCTा \\
\hline IFNG & TGTATTGCTITGCGTTGGAC & TGACCAGAGCATCCAAAAGA \\
\hline IFNGR2 & ATCAGCGATGTCAAAGGGAG & TGACAATGCCTTGGTITCAA \\
\hline IFNGR1 & TGGCATGATCTGGTACTCCC & CTTGTCATGCAGGGTGTGAG \\
\hline STAT1 & TGAATATTCCCCGACTGAGC & AGGAAGACCCAATCCAGATGT \\
\hline JAK1 & СTGCTCATTGTCGTTGGTTC & TGCCCTGTATGACGAGAACA \\
\hline GAPDH & AATCCCATCACCATCTTCCA & TGGACTCCACGACGTACTCA \\
\hline TNF & AGATGATCTGACTGCCTGGG & CTGCTGCACTITGGAGTGAT \\
\hline TGFB1 & AAGTTGGCATGGTAGCCCTT & CCCTGGACACCAACTATTGC \\
\hline ILIB & AAGCCCTTGCTGTAGTGGTG & GAAGCTGATGGCCCTAAACA \\
\hline PARK7 & GTGCAGTGTAGCCGTGATGT & CCTCCTGGAAGAACCACCAC \\
\hline SOD1 & GTGATTGGGATTGCGCAGTA & TGGTITGAGGGTAGCAGATGAGT \\
\hline UCP2 & CAGCCAGCGCCCAGTACC & CAATGCGGACGGAGGCAAAGC \\
\hline
\end{tabular}

\section{Western blot}

Samples were lysed in 1\% Triton X-100 (Sigma) in lysis buffer [100 mM $\mathrm{NaCl}, 50 \mathrm{mM}$ Tris- $\mathrm{HCl}, 1 \mathrm{mM}$ EGTA, $\left.10 \mathrm{mM} \mathrm{MgCl}\left(6 \mathrm{H}_{2} \mathrm{O}\right)\right]$, measured for protein concentration, loaded onto $4-12 \%$ gels (NuPage ${ }^{\circ}$ ), and processed as described [33].

\section{Electrophysiology}

Primary CNs from WT and Ifnb ${ }^{-/}$were cultured as described above. PIAS2 was overexpressed using AAV6 pAAV-SYN1-mCherry-mouse Pias2 or pAAV-SYN1-mCherry (Vector Builder) by $\mathrm{CaCl} 2$ method on day 1 of culture or knocked down by siRNA with the Accell SMART pool system (Thermo Scientific) for the last $72 \mathrm{~h}$ after 9-10 days in culture. SHSY5Y cells were kept in artificial cerebrospinal fluid, containing (in mM) $125 \mathrm{NaCl}, 2.5$ $\mathrm{KCl}, 26 \mathrm{NaHCO}_{3}, 2 \mathrm{CaCl}_{2}, 1 \mathrm{MgCl}_{2}, 1.25 \mathrm{NaH}_{2} \mathrm{PO}_{4}$, and 25 glucose, in $95 \% \mathrm{O}_{2}$ and $5 \% \mathrm{CO}_{2}$ at room temperature. Glass pipettes (borosilicate, resistance 5-10 M $\Omega$, Sutter Instruments, US) were filled with (in mM): $122 \mathrm{~K}$-gluconate, $2.5 \mathrm{MgCl}_{2}, 0.3 \mathrm{CaCl}_{2}, 5.6 \mathrm{Mg}$-gluconate, $5 \mathrm{~K}$-HEPES, $5 \mathrm{H}$-HEPES, $5 \mathrm{Na}_{2} \mathrm{ATP}, 1$ EGTA, 10 biocytine, Alexa Fluor $488(10 \mu \mathrm{M}$, Sigma), and $\mathrm{KOH}$ to adjust the $\mathrm{pH}$ to 7.4 .

Cells were visualized under a BW51WI microscope (Olympus, Japan). Whole-cell recordings were made in voltage clamp mode with a Multiclamp 700B amplifier (Molecular Devices, US). Recordings were sampled at $20 \mathrm{kHz}$ with a 16-bit analog-to-digital converter (DIGIDATA 1440; Molecular Devices, US) and displayed with Clampex 10.2 (Molecular Devices, US). Fast transient sodium inward currents [34] were evoked by depolarizing steps of 10-mV increments, applied from $-90 \mathrm{mV}$. The inward current amplitude (calculated as the difference between the peak and baseline) was maximal at 0 or $+10 \mathrm{mV}$. 


\section{Statistical analysis}

Data were analyzed by unpaired two-tailed Student's t-test, ANOVA (parametric) or Mann-Whitney $U$ (non-parametric); correlation analysis; Shapiro-Wilk normality distribution test and Fisher's exact test using Prism software. $P<0.05$ was significant. Error bars are \pm SEM.

\section{RESULTS \\ Neuronal IFN $\beta$-IFNAR signaling is defective and associated with increased PIAS2 in patients with sporadic Parkinson disease dementia by transcriptomic analysis}

Although rare familial genes that are associated with PD have been identified, the genes that have been linked to the etiology of SPD remain poorly characterized. We tested the hypothesis that the heterogeneous group of patients with SPD and SPDD will have defects in several genes in the same signaling pathways. To this end, we analyzed data from a previously published DNA microarray study on laser-captured CNs that were obtained from sPD patients with no dementia (sPDND) $(n=15)$, sPDD patients $(n=13)$ with clinical dementia, and HCs $(n=14)$ [25]. First, we performed class prediction to determine the core enriched genes that were differentially expressed between sPDD and sPDND patients. We found 638 such genes $(P$ value $\leq 0.01)$ that classified the two patient groups (Supplementary Data File 1). This signature (Fig. 1a) also allowed the distinction of Ifnb-deficient PD mice [9] (GSE63815) from WT controls, validating it as a relevant animal model for PD (Fig. 1b).

By GSEA of the patient dataset, the cytokine-cytokine receptor interaction pathway was the highest ranking Kyoto Encyclopedia of Genes and Genomes (KEGG) pathway that was associated with SPDD versus HCs and SPDND (Supplementary Table $1 a, b)$. This pathway was also significantly associated with sPDND patients compared with HCs (Supplementary Table 1c), indicating that defects in cytokine signaling intensify as the disease progresses to dementia. To verify these findings, we analyzed three additional independent studies on gene expression profiles in the SN from sPD patients $(n=45)$ and HCs $(n=$ 25): GSE7621 [35], GSE20141 [36], and GSE49036 [37]; the status of dementia was not indicated in the two former studies. When combining these studies, the cytokine-cytokine receptor interaction pathway was significantly regulated $[P \leq 0.001$; normalized enrichment score (NES): 3.5537] and ranked number 1 by GSEA, underscoring its importance in PD (Fig.1c and Supplementary Table 1d).

To verify these results, we measured the mRNA levels of select genes within the cytokine-cytokine receptor interaction pathway from the cortex of a separate in-house cohort of sPD patients and HCs. IFNG, IFNGR1, and STAT4 were highly dysregulated between HC, sPDND, and sPDD patients. IL1B, TNFA, and TGFB1 mRNA levels were significantly higher in SPDD versus the other groups (Fig. 1d), confirming that also in our cohort, disrupted cytokine signaling correlates with the progression of PD to dementia.

Consistent with these results, by GSEA of a neuronal microarray (GSE63815) from our Ifnb-deficient PD mouse model [9], the cytokine-cytokine receptor interaction pathway was significantly

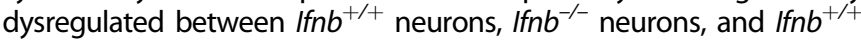
neurons that were treated with recombinant interferon-beta (rIFN- $\beta$ ), as confirmed in untreated versus rIFN- $\beta$-treated $/ \mathrm{fn}^{-/-}$neurons (Supplementary Table 1e-g). Next, we applied the gene signature of Ifn $b^{-/-}$versus Iffn $b^{+/+}$neurons and Ifn $b^{-/-}$versus rIFN- $\beta$-treated Ifn $b^{-/-}$ neurons in the cytokine-cytokine receptor interaction pathway to determine whether it could differentiate SPDD patients from $\mathrm{HCs}$ and PDND. The gene signatures from the $1 \mathrm{fn} b^{-/-}$PDD mouse model distinguished SPDD patients from the other two groups (Supplementary Fig. S1a, b), demonstrating that the PD mouse model [9] mimics the genetic and pathological aspects of PD dementia. Moreover, it highlights that the absence of Ifnb is sufficient to disrupt other cytokines and their signaling.
All of the top signaling pathways (cytokine-cytokine receptor, JAKSTAT, RIG, and Toll-like-receptor) contain several type I interferonsIFNAs and IFNB1 (and IFNAR) - and downstream molecules, such as JAKs, STAT2, and PIAS2 (Supplementary Table 1). To determine the relevance of defective neuronal IFN $\beta$-IFNAR signaling to PD, we generated a common type I IFN gene signature, based on the literature (Supplementary Data File 2), and compared it with patient datasets by GSEA. Heatmaps of the signature indicated that IFNAR1, $J A K s$, and STATs were transcriptionally upregulated in SPDD patients, as were negative regulators of this pathway, in particular PIAS2 (Fig. 1e). The type I IFN gene signature was significantly enriched in sPDD versus $\mathrm{HCs}(P \leq 0.001)$ and sPDND patients $(P \leq 0.001)$ (Fig. $1 f)$. When pooling the three cohorts GSE7621, GSE20141, and GSE49036, the type I IFN signature distinguished SPD from HCs by GSEA ( $P \leq$ 0.001, NES: 1.686) (Fig. 1g). The GSE49036 study separated PD patients with and without dementia [37]; thus, we used it to validate the previous dataset [25], and type I IFN-related pathways appeared among the top 20 most highly regulated pathways (Supplementary Table $1 \mathrm{~h}-\mathrm{j}$, marked in bold). Similarly, significant gene enrichment was observed in the sPDD versus SPDND and HC groups (Fig. 1h), verifying our previous findings.

Next, we generated an interactive signaling map of our type I IFN signature genes (PathoVisio), comparing PD patients and HCs from the pooled cohorts (GSE7621, GSE20141, and GSE49036). This analysis revealed that several genes, both activating and inhibiting genes, were differentially regulated-e.g., IFNAR genes were downregulated, whereas PIAS2, a negative regulator of STAT2, was upregulated in PD patients (Supplementary Fig. S1c)thus indicating that the signaling pathway as a whole is blocked. In support of these findings, Stat1 and Stat2 mRNA in cultured Ifn $b^{-/-}$neurons were unaffected compared with WT $\left(I f n b^{+/+}\right)$ neurons, but rIFN- $\beta$ treatment induced these genes (Fig. 1i). Notably, Pias2 mRNA was significantly upregulated in Ifn $b^{-1-}$ neuron cultures compared with Ifn $b^{+/+}$ones (Fig. 1i). Moreover, neuronal cells in hippocampal and cortex tissue expressed high levels of PIAS2 in the Ifnb ${ }^{-/-}$, Ifnar $1^{-/-}$, and nes ${ }^{\text {Cre }}:$ Ifnar ${ }^{\text {fl/fl }}$ (Ifnar1deficient only in neuroectodermal cells) PDD models (Fig. $1 \mathrm{j}$ and Supplementary Fig. S1d).

Next, we verified selected genes in the type I IFN signature in our patient cohort by RT-qPCR, which confirmed their differential regulation in SPD patients and those with dementia, including IFNA17, IFNB, and IFNAR1 (Fig. 1k). JAK1, STAT1, and STAT2 mRNA levels were unchanged, but IRF3 mRNA level was significantly higher and IRF9 one was downregulated in both SPD groups (Fig. 1k). IRF9 is an important factor in IFN- $\beta$-IFNAR signaling, in which IRF9 binds to phosphorylated STAT1-STAT2 heterodimers to generate the ISG3 complex, which in turn binds to DNA and regulates gene expression [38]. PIAS2 mRNA rose in SPDND but peaked dramatically in SPDD (Fig. 1k). Consistent with these data, $\beta_{\text {III-tubulin }}{ }^{+} \mathrm{CNs}$ had more intense PIAS2 staining in both SPD groups compared with HCs (Fig. 1I). Although IFNA17 and IFNAR1 were higher in some SPD groups, the defective IRF9 and increased PIAS2 in SPD patients suggested a block in type I IFN signaling. Furthermore, when Ifnarl or Ifnb was deleted in mice, neurons upregulated Ifnb and Ifnar2 mRNA, respectively (Fig. $1 \mathrm{~m}, \mathrm{n}$ ), reflecting their attempt to compensate for inadequate IFN $\beta$-IFNAR signaling.

These findings indicate that defects in IFN $\beta$-IFNAR-regulated signaling are linked to the development of SPD and its progression to dementia and that the heterogeneity in SPD is associated with differential expression of several genes in the type I IFN pathway. Although such genes might be differentially expressed between individuals with SPD and between early and later stages of the disease, they might cause the same outcome: blockade of IFN $\beta$-IFNAR signaling, thus accelerating disease progression. 


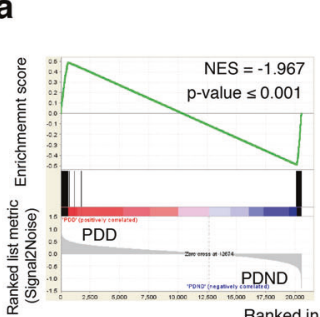

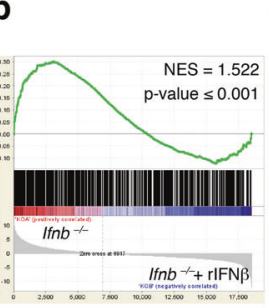

Tanked in Ordered Dataset

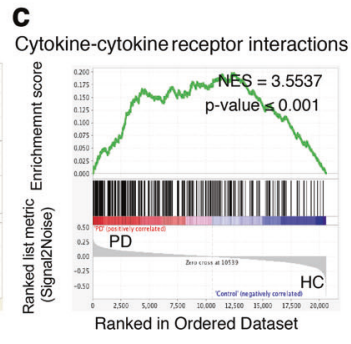

e

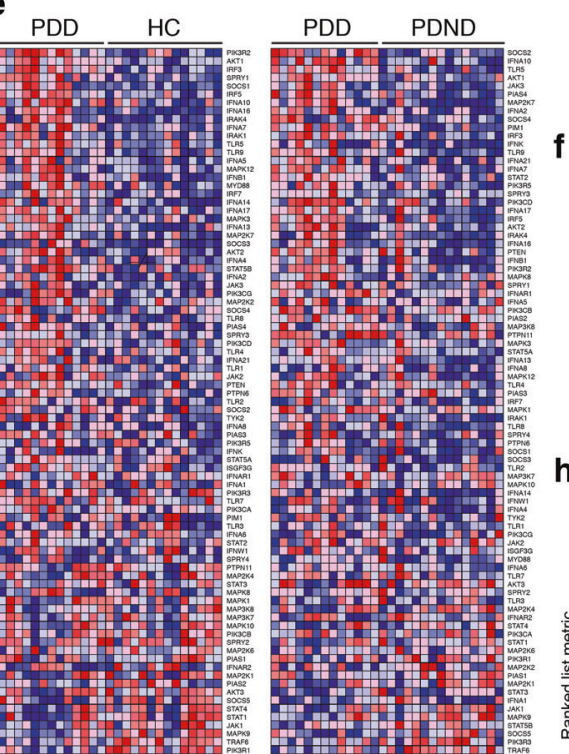

\section{f}
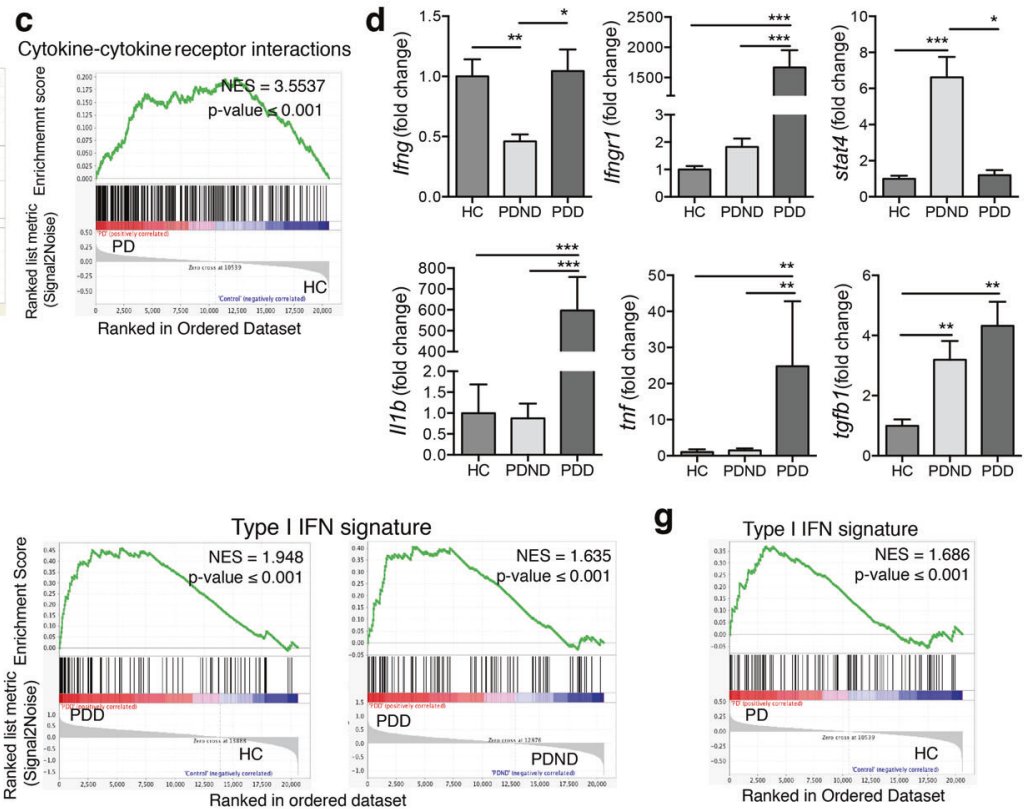

g
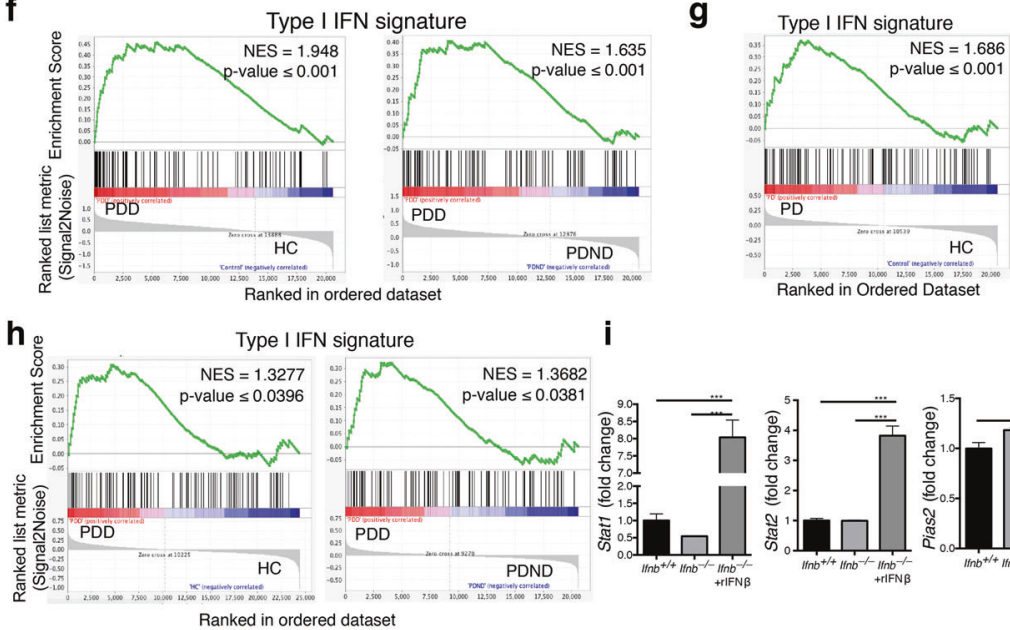

i
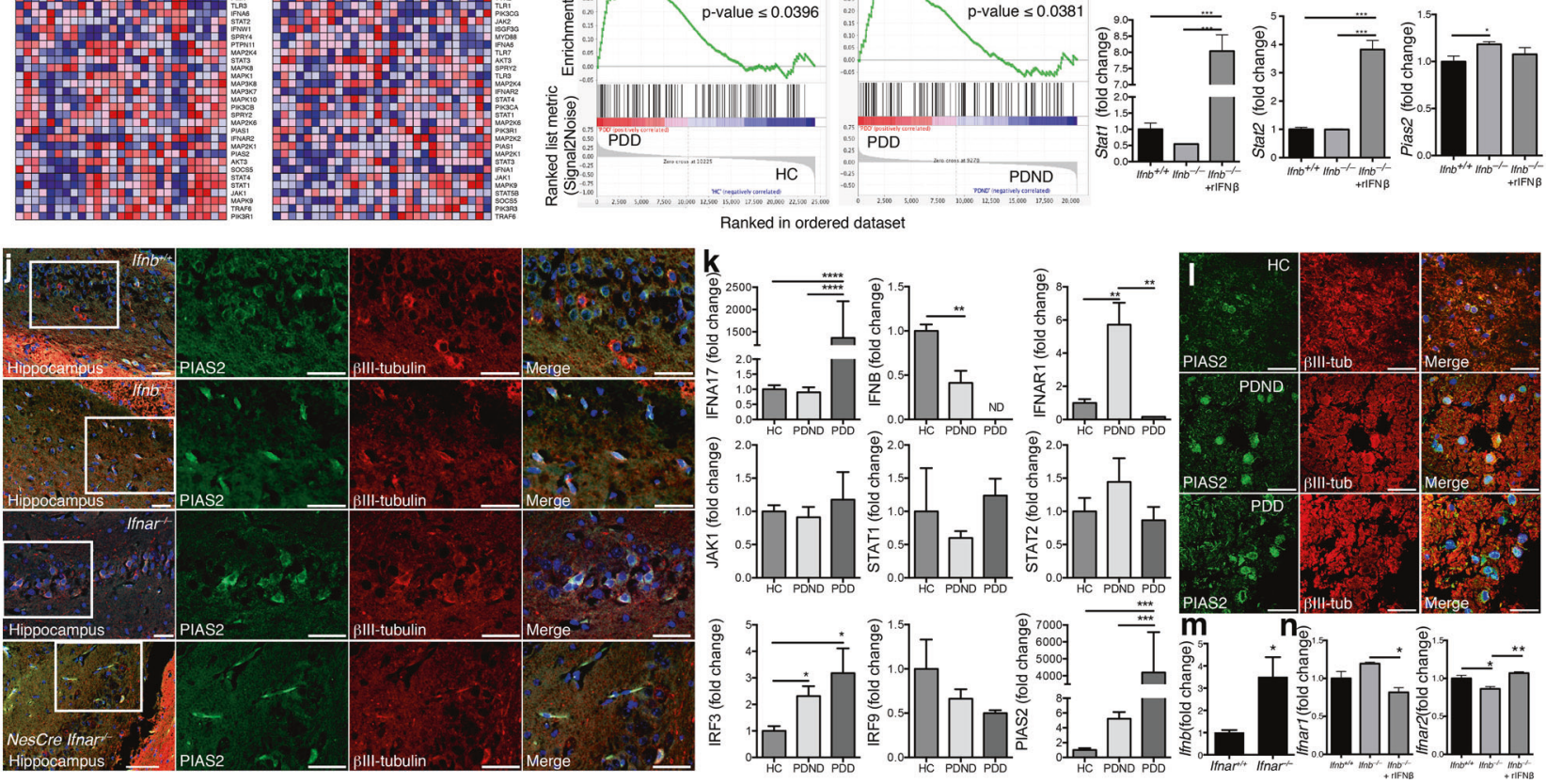

Fig. 1 Upregulation of neuronal PIAS2, a negative regulator of IFN $\beta$-IFNAR signaling, in the brains of sporadic PD patients. a GSEA class prediction analysis distinguishing sporadic Parkinson disease patients with dementia (sPDD; $n=13$ ) from sPD patients with nondementia (sPDND; $n=15$ ), used as the discovery cohort. b Core enriched gene signature (638 genes) of sPDD versus sPDND patients used as a class predictor of Ifnb ${ }^{-/-}$neurons with and without rIFN- $\beta$ treatment $(100 \mathrm{U} / \mathrm{ml}$ for $24 \mathrm{~h})$. c Enrichment plot of "cytokine-cytokine receptor interaction" pathway comparing microarrays from substantia nigra of PD patients $(n=45)$ and HCs $(n=25)$ pooled from three independent studies (GSE7621, GSE20141, and GSE49036) and used collectively as the discovery cohort. d RT-qPCR of medial frontal gyrus of HC, sPDND, and SPDD patients $\left(n=3-10\right.$ patients from in-house validation cohort). Graphs represent mean \pm SEM and ${ }^{*} P<0.05 ;{ }^{*} P<0.01 ;{ }^{* * *} P<0.001$ by one-way ANOVA and Turkey's post hoc correction test. e Heatmaps from GSEA of the type I IFN signature and comparing microarray data from sporadic Parkinson disease with dementia (sPDD; $n=13$ ), sPD with no dementia (PDND; $n=15$ ), and healthy controls (HC; $n=14$ ). f Enrichment plots of e. $\mathbf{g}, \mathbf{h}$ Enrichment plots of the type I IFN signature $\mathbf{g}$ comparing PDD with HC from the pooled cohorts GSE7621, GSE20141, and GSE49036. h Comparing PDD with PDND and HC from GSE49036. For all plots, NES: normalized enrichment score. i Foldchange in Stat1, Stat2, and Pias2 expression from microarray comparing Ifnb ${ }^{-/-}$and Ifnh ${ }^{+/+}$CGNs with or without rIFN- $\beta$ (GSE63815). Graphs represent mean \pm SEM of $n=3$ /group; ${ }^{*} P<0.05$ and ${ }^{* *} P<0.001$ by one-way ANOVA. $\mathbf{j}$ IF staining for PIAS2 (green), $\beta$ III-tubulin (red); and DAPI (blue) in hippocampus of WT, Ifnh ${ }^{-1-}$, Ifnar ${ }^{-/-}$, and nes ${ }^{\text {Cre }}$ :Ifnarr ${ }^{f / f l}$ mice. Bar, $50 \mu \mathrm{m}$. k RT-qPCR of selected genes from medial frontal gyrus regions of $\mathrm{HC}$, sPDD, and sPDND. Graphs represent mean $\pm \mathrm{SEM}, n=3-10 /$ group. ${ }^{*} P<0.05,{ }^{* *} P<0.01,{ }^{* * *} P<0.001$, and ${ }^{* * * *} P<0.0001$ by oneway ANOVA and Turkey's post hoc correction test. I IF staining for PIAS2 (green), $\beta$ III-tubulin (red), and DAPI (blue) in the frontal cortex region of HC, SPDD, and sPDND patients. Bar, $50 \mu \mathrm{m}$. $\mathbf{m ~ R T - q P C R}$ of Ifnb from brain of 3-month-old Ifnar $1^{-/-}$and Ifnar ${ }^{+/+}$mice. Graphs represent mean

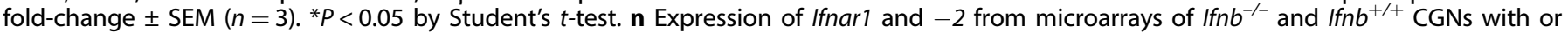
without rIFN- $\beta$ (GSE63815). Graphs represent mean \pm SEM of $n=3$ /group and ${ }^{*} P<0.05, * * P<0.01$ by one-way ANOVA. 

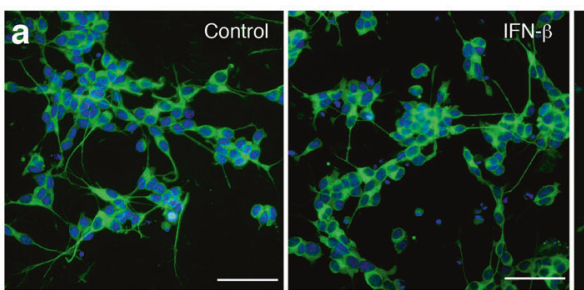

d

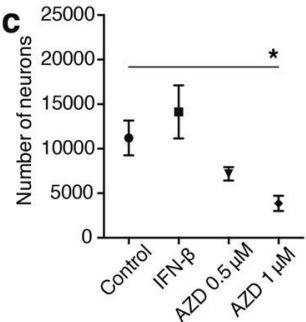

d PSTAT

pSTAT2

$\alpha$-tub $-0-0-0$

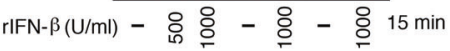

AZD $(\mu \mathrm{M})$ - - - $\stackrel{0}{0} \stackrel{0}{0} \stackrel{\circ}{\check{1}} 2 \mathrm{~h}$
AZZD $0.5 \mathrm{aMM}$

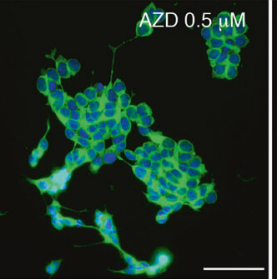

e

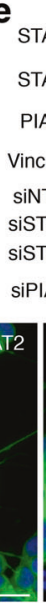

9
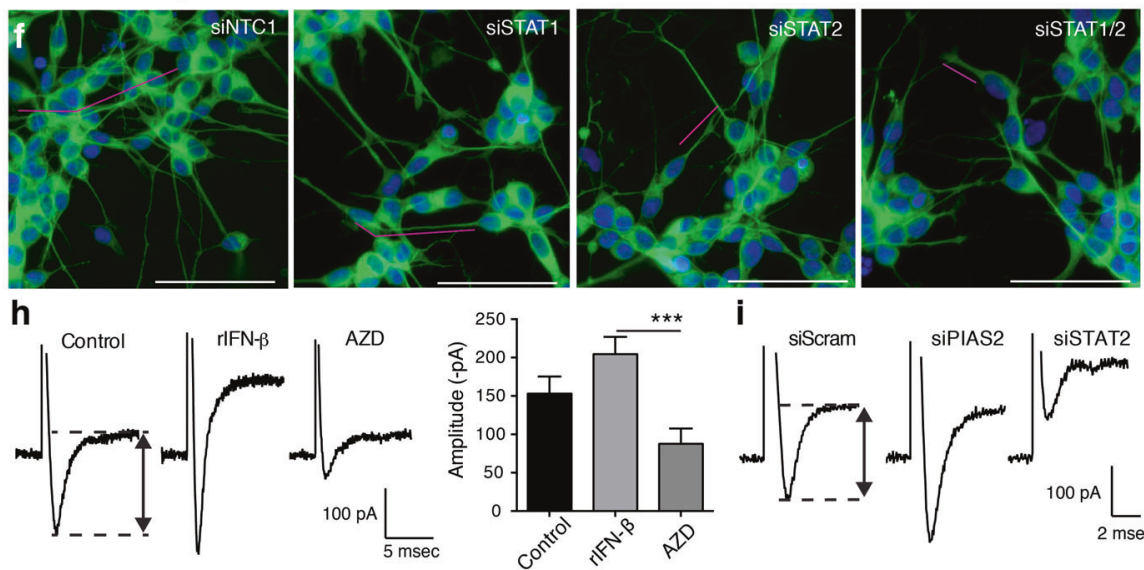

i siscram<smiles>C=C1CCCCC1C</smiles>

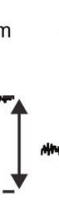

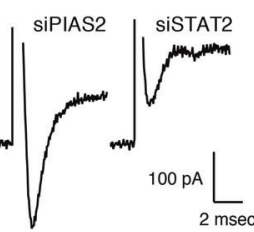

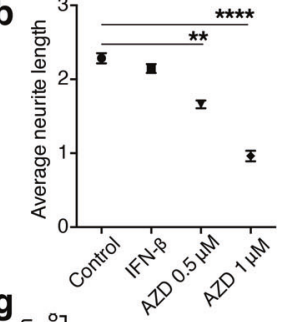

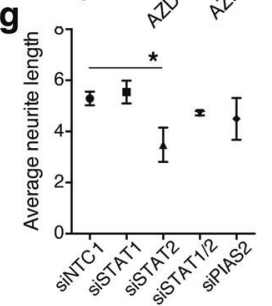

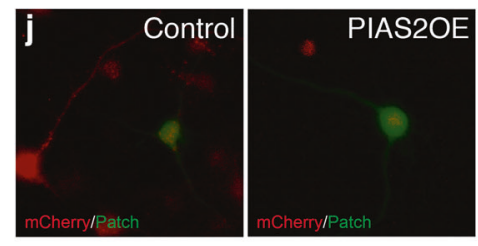

n

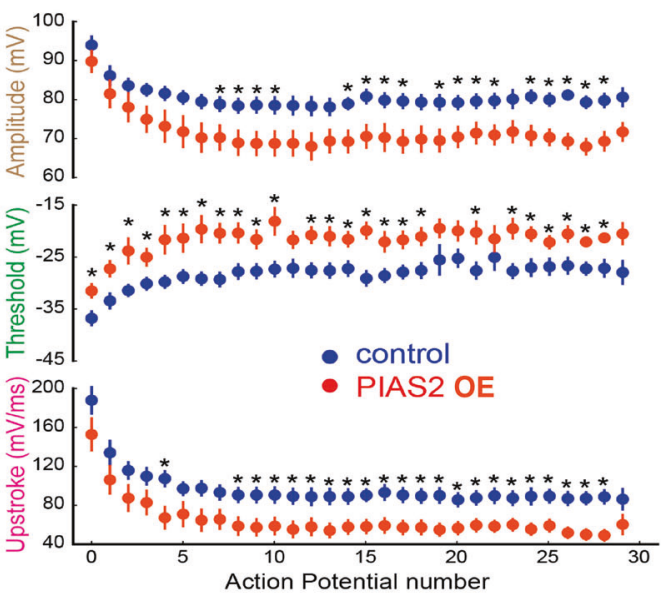

Potential number

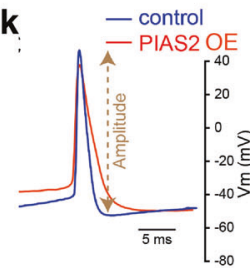

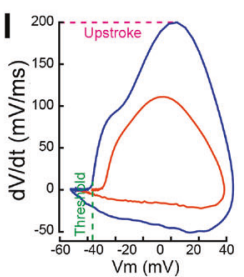

m
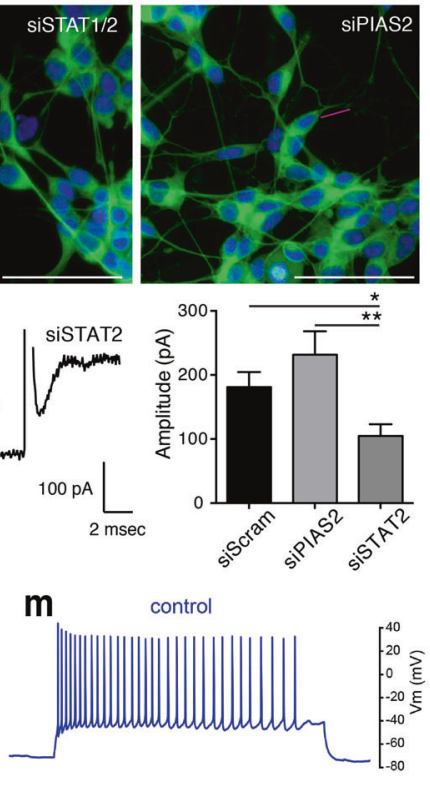

PIAS2OE
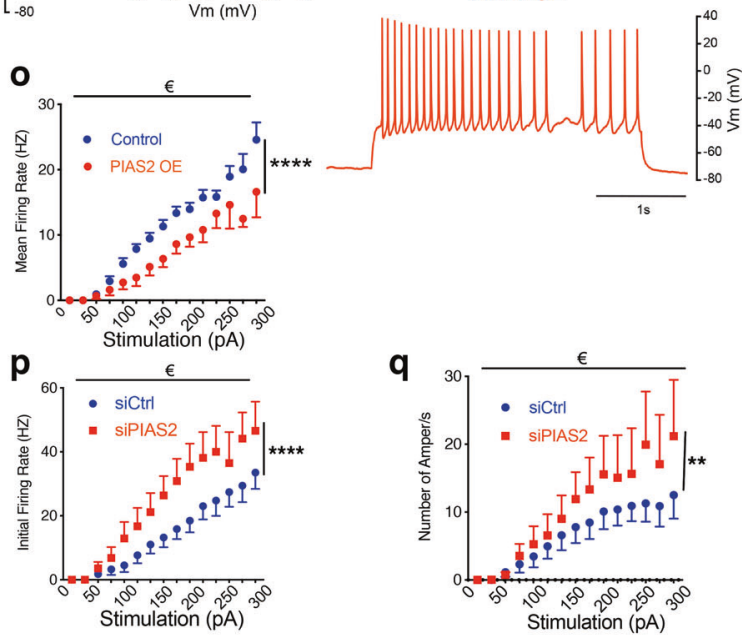

corrections for multiple comparisons [39, 40]. To determine whether gene sequence variants in IFN $\beta$-IFNAR signaling were associated with SPD, we analyzed a GWAS from the IPDGC, comprising 5333 PD cases and 12,019 controls with genotyped and imputed data on 7,689,524 SNPs [4]. As suggested [41], we 
Fig. 2 IFN $\beta$-dependent JAK-STAT2-PIAS2 signaling controls neuronal firing. a Representative IF images of differentiated SHSY5Y cells treated with rIFN- $\beta(1000 \mathrm{U} / \mathrm{ml})$ and AZD $(0.5$ or $1 \mu \mathrm{M})$ for $24 \mathrm{~h}$, showing $\beta$ III-tubulin (green) and DAPI (blue), b Quantified mean neurite length (estimated with $\beta$ Ill-tubulin), and c quantified neuronal survival (number of $\beta$ Ill-tubulin ${ }^{+} \mathrm{DAPI}^{+}$cells). d WB of differentiated SHSY5Y cells pretreated with AZD $(1 \mu \mathrm{M})$ for $2 \mathrm{~h}$ before 15 -min treatment with recombinant $(\mathrm{r}) \mathrm{IFN}-\beta(500-1000 \mathrm{U} / \mathrm{ml})$. e WB of siRNA KD in differentiated SHSY5Y cells, NTC means nontargeting control. f Confocal images showing neurite outgrowth with antibodies against $\beta$ III-tubulin, and g quantified neurite length. Bar, $50 \mu \mathrm{m}$, magenta bars underline one example neurite and graphs represent mean \pm SEM $(n=3)$. ${ }^{*} P<0.05$, ${ }^{* *} P$ $<0.01$, and ${ }^{* * *} P<0.0001$ by one-way ANOVA and Turkey's post hoc correction test. $\mathbf{h}$, i Curves represent amplitudes of the inward current evoked by a depolarizing voltage step in SHSY5Y cells after $\mathbf{h}$ treatment with rIFN- $\beta(1000 \mathrm{U} / \mathrm{ml}, 24 \mathrm{~h})$ or AZD $(0.5 \mathrm{uM}, 24 \mathrm{~h})$ or $\mathbf{i}$ siRNA knockdown of STAT2 or PIAS2. Bar graphs represent mean \pm SEM of $n=20$ individual cells (from two independent experiments). ${ }^{*} P<0.05$, $* * P$ $<0.01,{ }^{* *} P<0.001$ by Wilcoxon test. $\mathbf{j}$ Representative epifluorescence images illustrating primary cortical neurons transfected with control AAV mCherry or AAV PIAS2-mCherry (red). One mCherry-positive cell was patched in whole-cell configuration (green). $\mathbf{k}$ First action potentials generated by depolarizing current pulses in an AAV mCherry cell (blue) and AAV PIAS2-mCherry cell (orange). Note the smaller amplitude for the cell expressing AAV PIAS2-mCherry. I Phase plot representation of the action potentials. The PIAS2-mCherry expressing neurons had a higher threshold and a lower upstroke. $\mathbf{m}$ Examples of repetitive firing evoked in mCherry and PIAS2-mCherry expressing neurons. n-q Amplitudes, thresholds, and upstrokes measured during trains of 30 action potentials evoked by 3-s current pulses. Graphs represent mean \pm SEM. ${ }^{*} P<0.05$ by Mann-Whitney $U$ test and $€$ by two-way-ANOVA; ${ }^{* * * *} P<0.0001$ both raw and column factors $n=10$ for control and $n=11$ for PIAS2 from three independent experiments). Note the reduced firing frequency in PIAS2-mCherry expressing neurons.

assumed that SPD risk alleles were more likely to be distributed among genes with related functions.

Thus, we studied the enrichment of functionally related genes downstream of IFNAR: IFNAR1, STAT1, STAT2, JAK1, JAK2, AKT1, TYK2, SOCS1, SOCS3, and PIAS2. We identified 43 nominally significant sequence variants in PIAS2, JAK2, TYK2, and AKT1 (Table 1) that were associated with SPD patients. We also analyzed a recent PD GWAS update from the IPDGC, consisting of 7,893,274 SNPs from 13,708 PD cases and 95,282 HCs [28]. Although the accessibility analysis was more limited in this dataset, the number of PIAS2-related SNPs increased from 2 to 54 (Supplementary Data File 3), the raise could be due to an increase in genome coverage because of the availability of Haplotype Reference Consortium panel for imputation [42].

\section{Regulation of JAK-STAT2-PIAS2 is important for neurite outgrowth and neuronal survival and excitability}

To determine the function of IFN $\beta$-IFNAR-regulated proteins in neurons and their potential contribution to SPD pathology, we genetically and chemically modulated factors in this pathway. Using neuronally differentiated human SHSY5Y cells [43], we examined neuronal development and excitability by activating or blocking IFNAR/IFN- $\beta$ signaling chemically. AZD, a chemical blocker of JAK1/2, significantly reduced both average neurite length and neuronal survival (Fig. $2 a-c$ ). Human rIFN- $\beta$ treatment increased the phosphorylation of its immediate downstream target proteins, STAT1 and STAT2, which AZD blocked completely, thus validating it as a potent inhibitor of IFNAR-mediated signaling (Fig. 2d).

Next, we interfered with IFN- $\beta$ /IFNAR signaling by siRNA knockdown of STAT1, STAT2, and PIAS2 (Fig. 2e). As with AZD, neurite outgrowth was negatively affected by siRNA against STAT2 (Fig. 2f, g), and both approaches significantly inhibited neuronal firing, as evidenced by the lower amplitude of the transient inward current that was evoked by a depolarizing voltage step (Fig. $2 \mathrm{~h}$, i). Conversely, both rIFN- $\beta$ treatment and siRNA against PIAS2 significantly increased the amplitude compared with control neurons (Fig. $2 \mathrm{~h}$, i). In accordance, rIFN- $\beta$ has been reported to influence neuronal firing in neocortical pyramidal neurons from rat brain slices in a model of multiple sclerosis [44].

To verify the impact of PIAS2 on neuronal action potential, we established primary CNs that were infected by PIAS2-mCherry or mCherry-only-expressing AAVs, and mCherry-positive neurons were selected for patch clamp; positive penetration of the patch clamp was evidenced by injected of a green fluorescent marker (Fig. 2j). PIAS2-overexpressing neurons had a lower amplitude of the initial action potential, which correlated with a higher threshold and lower upstroke (Fig. 2k, I). When examining repeated action potential, PIAS2-expressing neurons had a significantly lower amplitude, higher threshold, and lower upstroke over time, and the mean firing rate was also lower with rising stimulation pulse intensities (Fig. $2 m-q$ ).

Our data support a crucial function for PIAS2 in neurons, downstream of IFN $\beta$-IFNAR- and STAT2-mediated signaling. Whereas IFN $\beta$-IFNAR signaling maintains neuronal homeostasis and proper neuronal excitability, its absence or disruption, particularly by PIAS2 overexpression, interferes with neuronal action potential, neurite outgrowth, and neuronal survival.

\section{PIAS2 overexpression alone in the brain is sufficient to cause PD-like dementia}

To determine the involvement of PIAS2 in SPD, WT, Ifn $b^{-/-}$, and Ifnar ${ }^{-1-}$ mice were injected with AAVs vectors that overexpress PIAS2-mCherry or mCherry-only under the neuron-specific SYN1 promoter in the SN and frontal cortex of the brain. Whereas WT mice that had been infected with mCherry-only AAV (CTR) performed significantly better over time in the cognitive (excluding memory) and motor learning tests-i.e., the Barnes maze and Rotarod tests, respectively-mice that overexpressed PIAS2-mCherry did not (Fig. 3a, b). As expected, mCherry-onlyinjected $/ \mathrm{fnb}^{-1-}$ and Ifnar $^{-1-}$ control mice did not improve with time, but PIAS2-mCherry overexpression did not aggravate the outcome further (Fig. $3 c$ and Supplementary Fig. S 2a-d), indicating that PIAS2 and the lack of Ifnb or Ifnar1 affect similar targets in this pathway. Moreover, the cognitive and motor declines due to PIAS2 overexpression in WT mice correlated with reduced TH mRNA and the loss of neurons, especially $\mathrm{TH}^{+}$ dopamine-producing neurons (Fig. $3 \mathrm{~d}$, e and Supplementary Fig. S2e).

Thus, PIAS2 overexpression in neurons is sufficient to cause motor and cognitive dysfunction, which is associated with the loss of dopaminergic neurons-the main hallmarks of PDD.

\section{Ectopic PIAS2 overexpression in the brain advances the} disease in a familial hSCNA-PD model

To determine the effects of PIAS2 overexpression in a human asyn (hSNCA)-induced familial model of PD, WT mice were injected unilaterally into the nigrostriatal region (SN and STR) with mCherry-only AAV (CTR) and PIAS2-mCherry or an inactive PIAS2 ${ }_{C 362 A}$-mCherry mutant (mutPIAS2), which carries a mutation in the RING finger-like domain [45], together with hSNCA (Fig. 3f). Compared with mutPIAS2, PIAS2 caused significantly greater motor impairments, based on motor coordination tests, as shown in climbing with a delay to climb and an increase of rearing time and in gait test where footprint showed variations in strides, 30 days after the nigrostriatal injections (Fig. $3 \mathrm{~g}-\mathrm{k}$ and Supplementary Fig. S2f). This was accompanied by a severe 

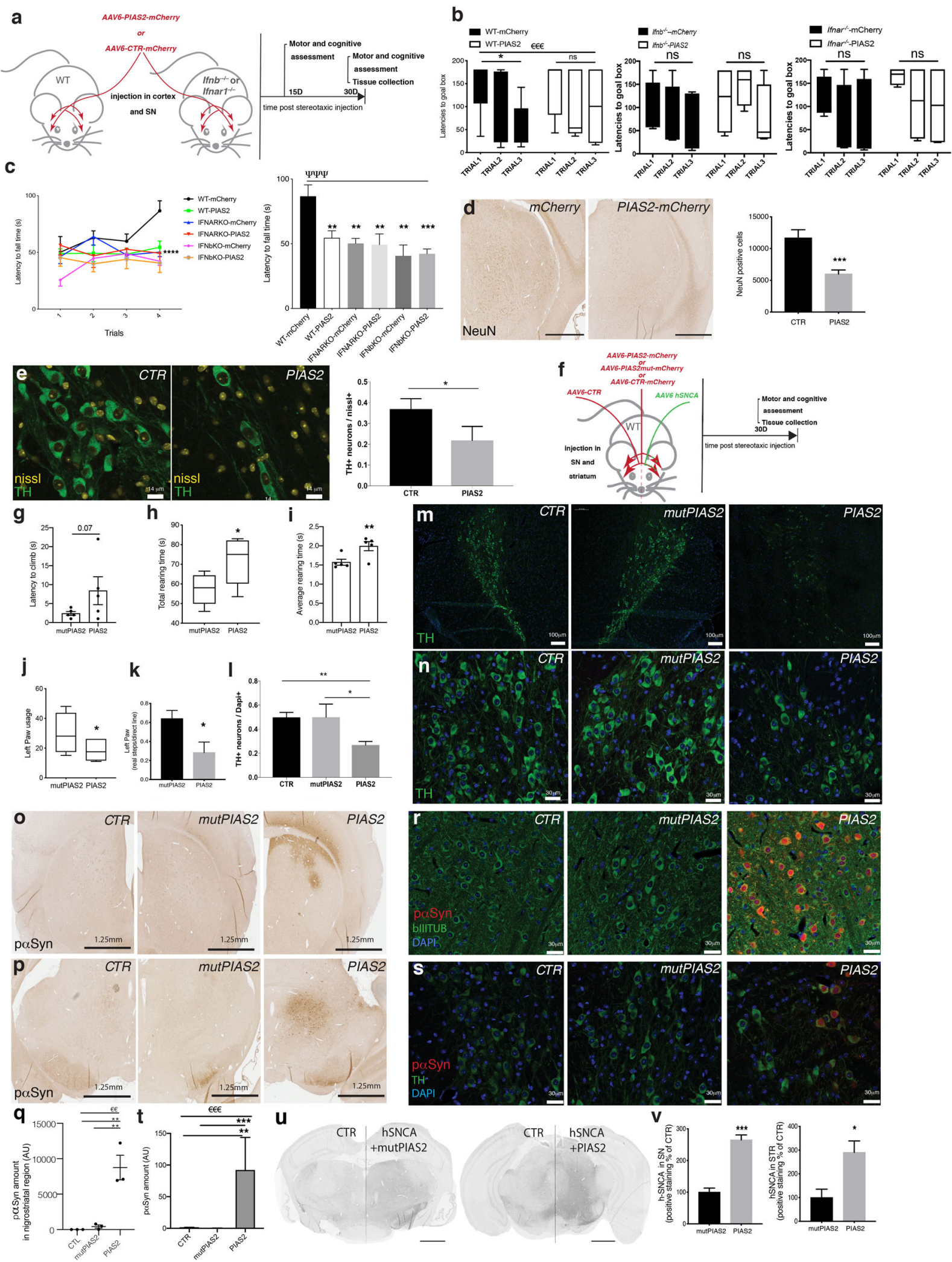

neurodegeneration and loss of dopamine-producing $\left(\mathrm{TH}^{+}\right)$ neurons (Fig. 3I-n). PIAS2-expressing mice experienced significant accumulation of pathogenic native phosphorylated a-syn (Fig. 3o-t) and hSNCA (Fig. 3u, v and Supplementary Fig. S2g-k) versus mutPIAS2.

These data establish that neuronal overexpression of PIAS2 in the familial hSCNA-PD model promotes the clinical and pathological manifestations of PD; accumulation of pathological forms of phosphorylated a-syn and reduction in the number of dopaminergic neurons. 
Fig. 3 Overexpression of PIAS2 alone in neurons is sufficient to cause neurodegeneration, motor and cognitive impairments, and PD-like dementia in mice. a Schematic of the experimental design. WT, Ifnb ${ }^{-/}$, and Ifnar ${ }^{-1-}$ mice injected with AAV6 PIAS2-mCherry or AAV6 CTR (mCherry) into the substantia nigra and cortex. Tests were performed at 15 and 30 days post injection. $\mathbf{b}$ Barnes maze test. Data in seconds \pm SEM. ${ }^{\in € \in} P<0.001$ by two-way ANOVA; ${ }^{*} P<0.05$, ${ }^{* *} P<0.01$ by Student's $t$-test. c Rotarod motor coordination test. Data in seconds \pm SEM; ${ }^{\Psi \Psi \Psi} P$ $<0.001$ by two-way ANOVA between groups and ${ }^{*} P<0.05,{ }^{* *} P<0.01,{ }^{* *} P<0.001$ by post hoc Dunnett's multiple comparisons test. $n=6 /$ WTmCherry CTR, 7/WT-PIAS2-mCherry, and $n=4 /$ each mCherry CTR- and PIAS2-mCherry-injected in Ifnb ${ }^{-/-}$and Ifnar $1^{-/-}$mice; total $n=29$. d NeuN IHC of the nigrostriatal region of brain of WT mice injected with AAV6 PIAS2-mCherry or AAV6 CTR mCherry and respective quantification. Data presented as number of positive cells \pm SEM. ${ }^{* * *} P<0.001$ by Student's $t$-test. $n=9 /$ CTR mCherry and $12 /$ PIAS2-mCherry. e IF for tyrosine hydroxylase (green) in SN in WT mouse brain injected with AAV6 PIAS2 or AAV6 CTR (mCherry) and respective quantification. Neurons stained with Nissl. Data on graph represent the number of $\mathrm{TH}^{+}$neurons \pm SEM, ${ }^{*} P<0.05$ by Student's $t$-test. $n=7 / C T R$ and $6 / P I A S 2$. f Schematic of the experimental design in a familial model of PD. WT mice unilaterally co-injected into the nigrostriatal region with AAV6 empty control and mCherry control on one side and human $\alpha$-syn (hSNCA) together with either PIAS2-mCherry or AAV6 mutPIAS2-mCherry on the other side. $\mathbf{g}$-i Motor coordination by climbing test at 16 days post injection: $\mathbf{g}$ latency to climb, $\mathbf{h}$ total rearing time, and $\mathbf{i}$ average rearing time. Data are in seconds $\pm \mathrm{SEM},{ }^{*} P<0.05,{ }^{*} P<0.01$ by unpaired Student's $t$-test. $\mathbf{j}$ Motor coordination by cylinder test at 30 days post AAV6 hSNCA/AAV6 PIAS2-mCherry or AAV6 hSNCA-mutPIAS2-mCherry injections showing left forepaw usage. Data in seconds \pm SEM; $n=6 /$ group. ${ }^{*} P<0.05,{ }^{*} P<0.01$ by unpaired Student's $t$-test. k Motor performance by gait test at 30 days post AAV6 hSNCA/AAV6 PIAS2-mCherry or AAV6 hSNCA/AAV6 mutPIAS2 injections; left paw locomotion analysis by footprint. Data in $\mathrm{cm} \pm \mathrm{SEM},{ }^{*} P<0.05,{ }^{* *} P<0.01$ by unpaired Student's $t$-test. $n=6 /$ group. I Quantification of $\mathbf{m}$, $\mathbf{n}$ IF staining of $\mathrm{TH}^{+}$(green) in the substantia nigra. Nuclei were stained with DAPI (blue); data in graph represent the number of TH-positive neurons \pm SEM. ANOVA ${ }^{*} P<0.05$, and ${ }^{* *} P<0.01$ by post hoc correction for multiple tests. $n=3$. o, $\mathbf{p}$ IHC of phosphorylated (p) $\alpha$-syn in brain of mice injected with AAV6 hSNCA/AAV6 PIAS2-mCherry or AAV6 hSNCA/mutPIAS2mCherry, $\mathbf{0}$ STR, and $\mathbf{p}$ SN and q quantification. Scale bar is $1.25 \mathrm{~mm}$. Data are mean $\pm \mathrm{SEM} ; n=3$. ${ }^{\epsilon} P<0.01$ by ANOVA and ${ }^{* *} P<0.01$ by post hoc correction with Dunnett's multiple correction test. $\mathbf{r}$, s IF of p $\alpha$-syn (red) and $\beta$-III-tubulin (green) in SN (r) and (s) with TH staining in SN. Nuclei were stained with DAPI; $\mathbf{t}$ quantification. Data in graph represent the number of p $\alpha$-syn-positive neurons \pm SEM of $n=3$; ${ }^{\epsilon \in \epsilon} P<0.001$ by ANOVA and ${ }^{* *} P<0.01$ and ${ }^{* * *} P<0.001$ by post hoc correction for multiple tests, applied to log-normal distribution. u IHC of hSNCA in SN and STR of mice injected with AAV6 hSNCA/AAV6 PIAS2-mCherry or AAV6 hSNCA/mutPIAS2-mCherry. Scale bars, $1 \mathrm{~mm}$. v Quantification of IHC of hSNCA in SN (left) and STR (right). Bars show normalized mean percentage of control \pm SEM for $n=4 ;{ }^{*} P<0.05$, *** $P<0.01$ by unpaired Student's $t$-test.

\section{Ectopic PIAS2 overexpression in neurons blocks mitophagy, triggers mitochondrial accumulation and oxidative stress, causing oxDJ1 to inhibit ERK1/2-P53 activity}

Next, we examined the molecular pathways by which PIAS2 affects neuronal function in vivo and in vitro. PD pathology is associated with mitochondrial dysfunction and oxidative stress, prompting us to assess these parameters further [46, 47]. Mitochondria accumulated significantly in neurons when PIAS2-mCherry was overexpressed (OE) in vivo in the brain alone or with hSCNA; this was associated with the build-up of phosphorylated a-syn (Fig. 4a-d and Supplementary Fig. S2i, j).

Rare mutations in DJ1 (PARK7) and loss of its function cause familial PD [48]. DJ1 regulates transcription and is an antioxidative stress sensor of mitochondria [49]; furthermore, it is a target of PIAS2 [50]. Consistent with these findings, PIAS2-mCherryOE in WT mice was sufficient to induce oxidative stress, as evidenced by the elevated levels of $80 \mathrm{HdG}$ (Fig. 4e), affecting mtDNA (mitochondrial DNA) oxidization as $80 \mathrm{HdG}$ colocalizes with mitochondrial protein Coxl (Fig. 4f). In support, PIAS2-mCherryOE in the brain also increased levels of oxidative (ox)DJ1 (Fig. $4 \mathrm{~g}, \mathrm{~h}$ ) and mRNA for ucp2 and park7 (the gene name for DJ1) but not sod1 (Supplementary Fig. S2I). These findings were verified in primary CN cultures. PIAS2 expression in CNs, reflected by increased levels of PIAS2-mCherry, effected mitochondrial accumulation, upregulated oxDJ1 and phosphorylated a-syn (Fig. 4i-k).

We have reported previously that lack of neuronal type I IFN signaling is causing autophagy block, and thereby resulting in intracellular pa-syn accumulation [9], and here we observed mitochondrial accumulation in brain and $\mathrm{CN}$ (Fig. 4a-d and Supplementary Fig. S2m, n) and increased oxDJ1 as a consequence of PIAS2OE (Fig. $4 \mathrm{~g}-\mathrm{k}$ ). We therefore investigated if PIAS2OE was dysregulating mitophagy, a selective autophagy process that removes damaged mitochondria. Consistent with mitophagy block, we observed that autophagy/mitophagy marker LC3II and LC3II/I ratio were increased (Supplementary Fig. S2O, p) and mitophagy specific marker Pink1 and mitophagy adaptor optineurin (optin) were significantly elevated in the brain of PIAS2OE mice (Fig. 4I-n).

Oxidative stress is reported to induce ERK-P53 signaling pathway [51] that is also shown to play a distinct role in regulating cell-cycle and death [52]. Moreover, ERK and nuclear P53 activation are reported to induce cellular autophagy [53]. We indeed identified P53 pathway as a result of active IFN $\beta$ induced signaling in neurons (Supplementary Table 1f), and it was also among the top 6 significantly dysregulated KEGG pathways between PDD and HC (Supplementary Table 1h), as well as the top 20 in PDND vs. HC (Supplementary Table 1i). Finally, we investigate how PIAS2-mCherry overexpression in mouse brain might impact activation of ERK $1 / 2$ and P53 signaling pathway. We found that the rise in oxDJ1 (Fig. $4 \mathrm{~g}-\mathrm{k}$ ) was negatively associated with activated/phosphorylated (p)ERK1/2 and pP53 in the brain of PIAS2OE mice (Fig. 4o, p). Of note, neurotoxicity and mitochondrial dysfunctions were not induced by mutPIAS2 control, despite a moderate but not significant impact of PIAS2 on sumoylation, SUMO1 levels (Supplementary Fig. S2q).

In conclusion, overexpression of PIAS2 in neurons causes pathology by triggering mitophagy block resulting in damaged mitochondrial accumulation and elevated oxidative stress, followed by increased oxidation of DJ1 and in turn inhibiting ERK1/2 and $\mathrm{P} 53$ by reducing their phosphorylation.

\section{PIAS2 knockdown rescues Parkinson disease-like dementia in Ifnb $^{-1-}$ mice}

To determine whether PIAS2 is a major driver of the clinicopathological manifestation of PDD in $/ \mathrm{fnb}^{-/-}$mice, we injected them with AAV that expressed siRNA against PIAS2 under the neuron-specific SYN1 promoter, into the nigrostriatal region and frontal cortex, and performed behavioral tests 16-30 days later (Fig. 5a). This resulted in about 50\% reduction of PIAS2 expression in the injected regions (Fig. 5b, c) and a moderate although not significant SUMO1 reduction (Supplementary Fig. S3a). In contrast to control siRNA (siCtrl)-injected Ifnb ${ }^{-1-}$ mice, Pias2 knockdown animals improved significantly in motor abilities (Fig. 5d) as shown by rotarod tests, and in cognition as shown by Barnes maze test over three trials (Fig. 5e). Consistent with this finding, they spent more time in zone 1, where the escape exit was placed on probe Days 2 (Fig. 5f) and 15 (Fig. 5g, h), indicating cognition improvements in short-term and long-term spatial memory. In vivo knockdown of Pias2 preserved the number of NeuN-positive (Fig. 5i, j) and 

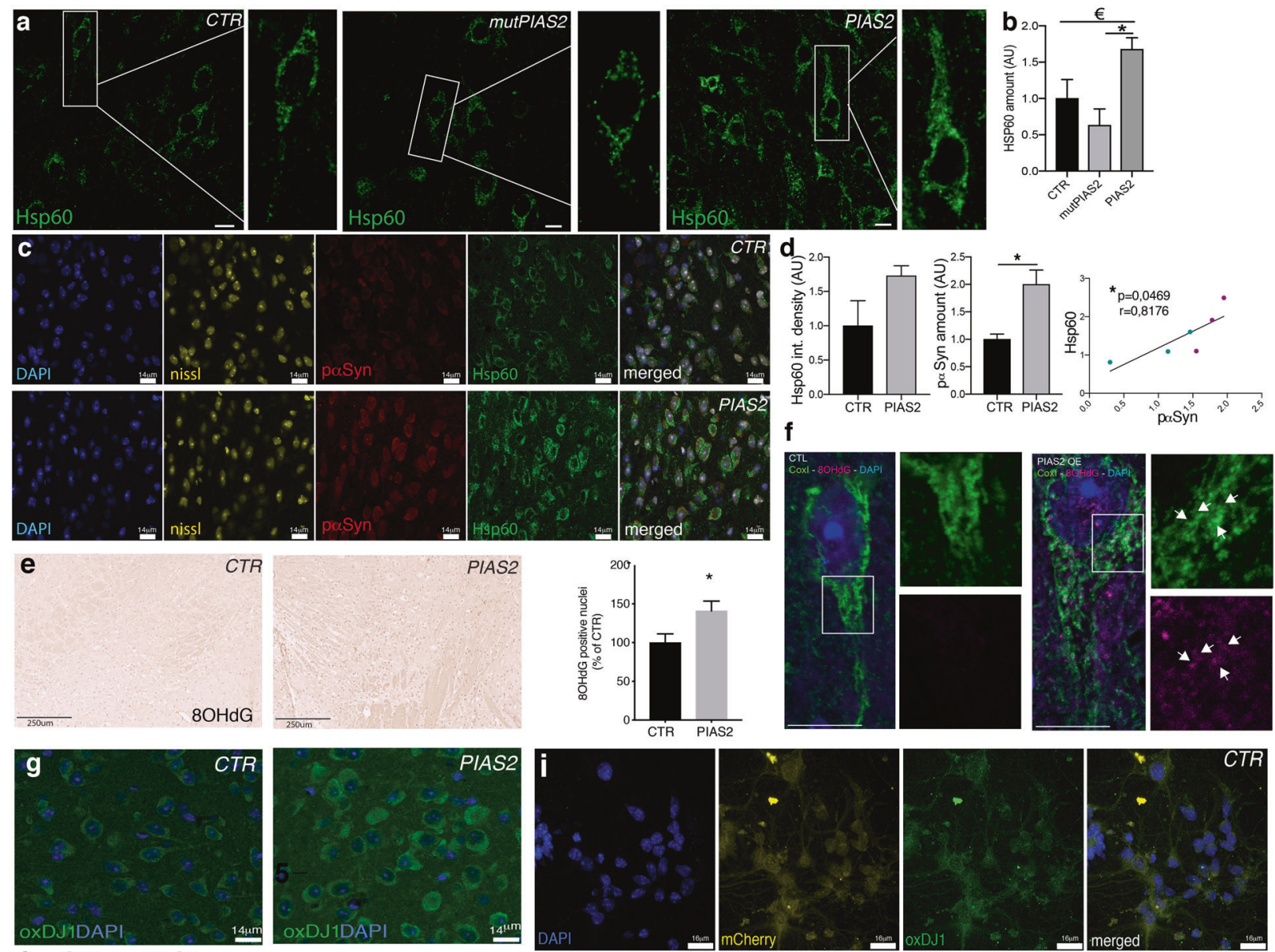

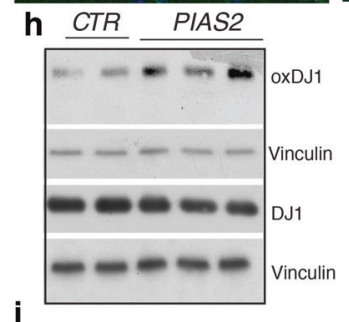

j
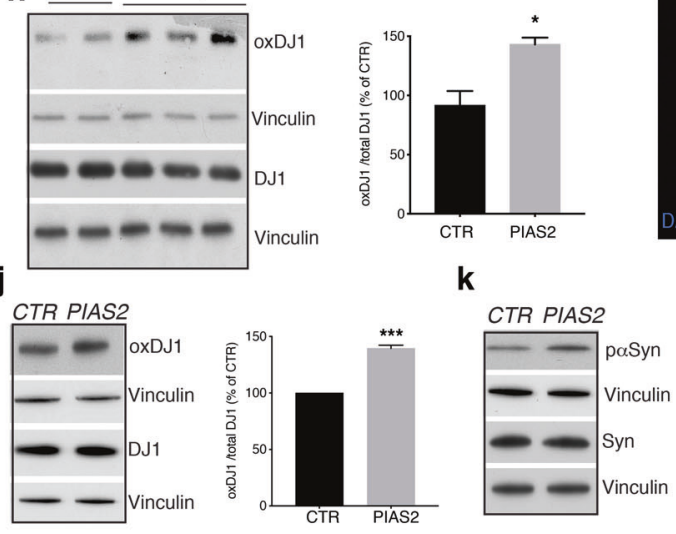

$\mathbf{k}$
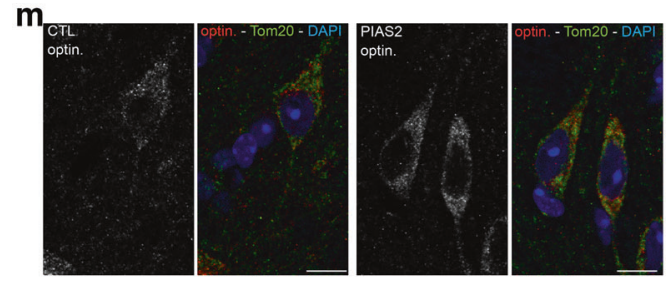

n

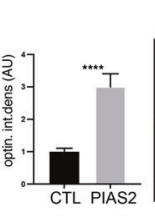

$\mathbf{0}$

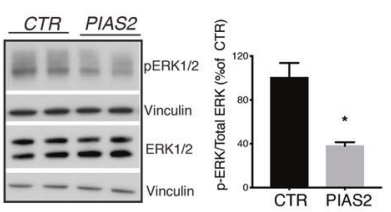

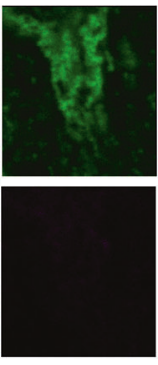
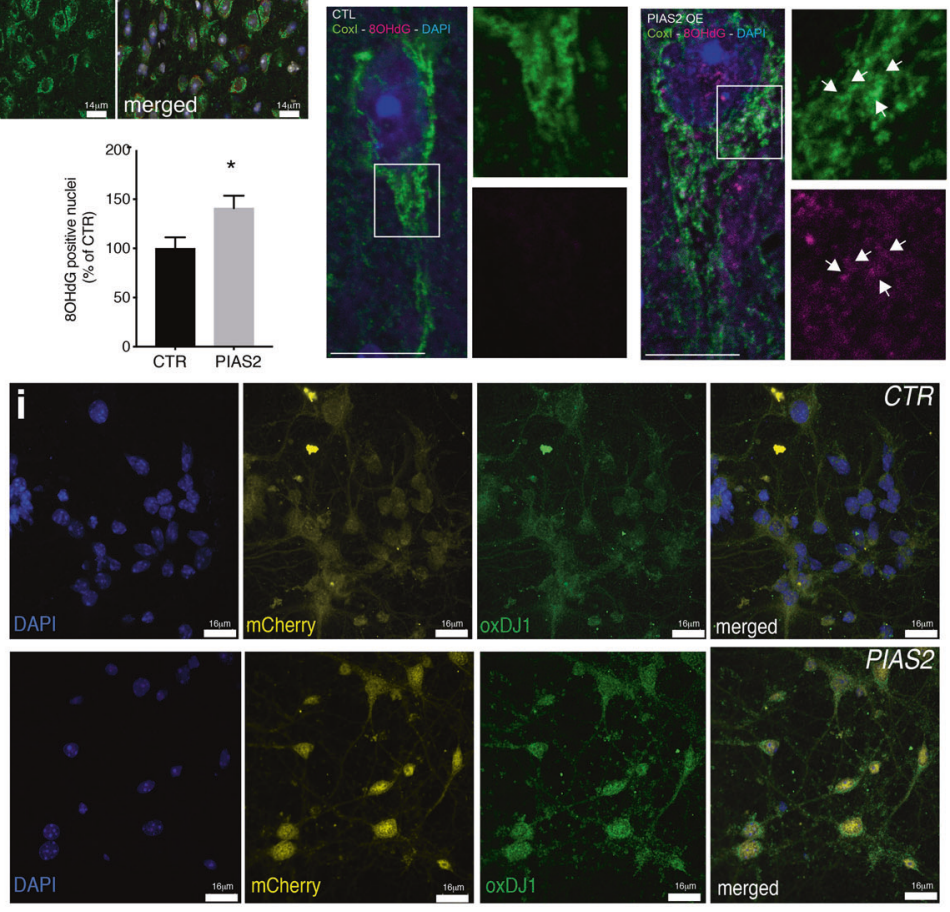

I
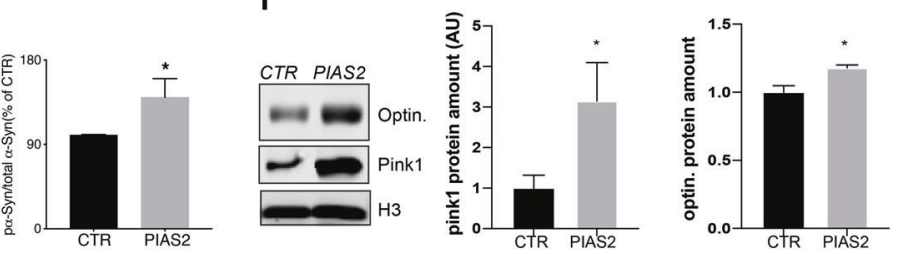

p

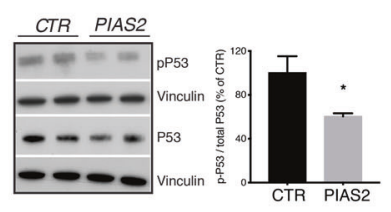

TH-positive dopamine-producing neurons (Fig. 5k, I) in the SN. Comparable results were obtained in the cortex where the significant reduction in PIAS2 was associated with decline in the intracellular pa-syn (Fig. $5 \mathrm{~m}-\mathrm{q}$ ).
These data verify that high neuronal PIAS2 levels correlate with cognitive impairments, similar to their association with dementia in PDD patients, and that eliminating neuronal PIAS2 reverses these deficiencies and neurodegenerative processes. 
Fig. 4 Ectopic expression of PIAS2 leads to dysregulated pP53, mitophagy block, mitochondrial accumulation, and oxidative stress. a IF of Hsp60 (green) and DAPI in AAV6 hSNCA/AAV6 PIAS2-mCherry- or AAV6 hSNCA/mutPIAS2-mCherry or CTR (mCherry) injected unilaterally into mouse brains. Scale bar is $14 \mathrm{um}$. b Quantification of Hsp60. Bars show mean \pm SEM of $n=3 ;{ }^{\epsilon} P<0.05$ by ANOVA and ${ }^{* *} P<0.01$ by post hoc correction for multiple tests. c, d IF of Hsp60 (green), phosphorylated (p) $\alpha$-Syn (red), nissl (yellow), and DAPI (blue) in brain of mice injected with AAV6 PIAS2-mCherry or AAV6 mCherry control. Scale bar is 14 um (c). d Quantification of Hsp60 (left) and po-syn (right) and correlation between Hsp60 and po-syn (below). Bars show mean \pm SEM; ${ }^{*} P<0.05$ by unpaired Student's $t$-test. $n=3 / g$ roup. Pearson's correlation/simple linear regression test, $n=9 /$ group; mCherry controls are in turquoise and PIAS2-mCherry is in purple. e IHC and quantification of $8 \mathrm{OHdG}$ in brains of WT mice overexpressing PIAS2-mCherry or CTR mCherry. Data represent mean number of positive nuclei and are presented as $\%$ of control. Scale bar, $250 \mu \mathrm{m}$. ${ }^{*} P<0.05$ by unpaired Student's $t$-test; $n=6 / g$ roup. f IF for Tom 20 and $80 H d G$ in striatum of WT mice overexpressing PIAS2-mCherry or CTR mCherry. Arrows indicate colocalization regions of $80 \mathrm{OH}$ G and Coxl. Scale bars equal 10 microns. g IF for oxidized DJ1 in WT mice brain overexpressing mCherry or PIAS2-mCherry. Nuclei stained with DAPI. Scale bar 14 um. h WB of oxDJ1 and total DJ1 for CTR and PIAS2 expression. Vinculin was used as a loading control. Data represent \% of control \pm SEM of $n=3$; ${ }^{* * *} P<0.001$ by Student's $t$-test. i Expression of AAV6 PIAS2-mCherry or CTR (mCherry/yellow) in primary cortical neurons (CN) and IF staining for oxidized DJ1 (green) and nuclei stained with DAPI (blue). Scale bar, $16 \mu \mathrm{m}$. j, k WB of $\mathbf{j}$ oxidized DJ1 and total DJ1 and $\mathbf{k}$ p $\alpha$-syn and total $\alpha-$ syn .k in CNs overexpressing PIAS2-mCherry or mCherry CTR. Vinculin was used as a loading control. Data represent $\%$ of control \pm SEM; ${ }^{*} P<$ 0.05 by Student's $t$-test. $n=3$. I WB for optin. and PINK1. H3 is used as a loading control. Data represent $\%$ of control \pm SEM, ${ }^{*} P<0.05$ by Student's $t$-test. $n=3$. $\mathbf{m}$ Staining for optin. and Tom20. $\mathbf{n}$ Quantification of I. WB of, o pERK1/2 and total ERK $1 / 2$, and $\mathbf{p}$ pP53 and total P53 in WT mouse brains overexpressing PIAS2-mCherry or mCherry CTR. Vinculin is used as a loading control. Data represent $\%$ of control \pm SEM, ${ }^{*} P$ $<0.05$ by Student's $t$-test. $n=3-4$.

\section{PIAS2 knockdown reverses PDD pathology by preventing mitochondrial oxidative stress and restoring pERK1/2- pP53 signaling}

Next, we examined whether siRNA-mediated knockdown of Pias2 in the brain of Ifnb ${ }^{-/-}$mice mitigates the signals that contribute to mitochondrial pathology. siPIAS2 enhanced ERK1/2 activationpERK $1 / 2$ increased and translocated primarily to the nucleus, and the pERK/ERK ratio rose significantly (Fig. 6a, b). Similarly, knockdown of PIAS2 upregulated pP53 in neurons (Fig. 6c, d), indicating the reversal of the defects that are observed in Ifn ${ }^{-1-}$ neurons.

The pathology of PD is linked to greater oxidative stress [54], contributing to the progressive loss of neurons. Accordingly, in our PDD model, the control Ifn $b^{-/-}$group accumulated damaged mitochondria, with high levels of $8 \mathrm{OHdG}$, in particular affecting mtDNA, and DJ1 oxidation (Fig. 6e-i and Supplementary Fig. S3b, c). The Pias2 knockdown lowered the mitochondrial mass (Fig. $6 \mathrm{e}$ and Supplementary Fig. S3b, c), the amount of optin (Fig. 6f), and the oxidative marker $8 \mathrm{OHdG}$ colocalized with Coxl (Fig. $6 \mathrm{~g}$, h and Supplementary Fig. S3b), and the ratio between DJ1 and oxidized DJ1 (Fig. 6i and Supplementary Fig. S3C). Because it has been reported that CNs in culture express $\mathrm{TH}$ [55], these results were verified in primary If $\mathrm{fb}^{-/-} \mathrm{CN}$ cultures, in which knockdown of PIAS2 upregulated TH in Ifnh ${ }^{-/-}$neurons (Fig. 6k) and significantly increased pERK $1 / 2$ and pP53 levels (Fig. $6 \mathrm{l}-0$ ). These events in turn prevented the excess in oxidative stress, as evidenced by the decrease in oxDJ1, 8OHdG, and mitochondrial accumulation (Fig. 6p-s).

Collectively, our results demonstrate that high and persistent PIAS2 expression is an important driver of the PD-like pathology that is observed in Ifn $b^{-/-}$mice. Conversely, siRNA against PIAS2 restores the activity of pERK1/2-pP53 and the anti-redox activity of DJ1, preventing oxidative damage and mitochondrial dysfunction. These findings also reinforce that in vivo high expression of PIAS2 induces cognitive impairments and its knockdown improves cognition in the Ifn $b^{-/-}$PDD model (Supplementary Fig. S4).

\section{DISCUSSION}

Our aim in the current study was to recognize signaling pathways that are disrupted in SPD and its progression to dementia in SPDD. By transcriptomic analysis, we first examined the gene expression profiles of SPD patients by GSEA. By combining four independent transcriptomic datasets from SPD patients to create a discovery cohort, we found that the cytokine-cytokine receptor and its related JAK-STAT pathways were the most highly dysregulated pathways compared with healthy individuals. Moreover, to narrow the pathway, we identified strong association of SPD with dysregulated type I IFN signaling among the cytokine-cytokine receptor pathway. Furthermore, these data cement the relevance of defective type I IFN signaling to SPD, especially SPDD, given our findings that the lack of Ifnb or Ifnar 1 in mice [9] leads to clinical features that resemble SPDD. Our additional analysis revealed that select genes in type 1 IFN signaling have a robust link to SPD and dementia. While some individual proteins involved in the type I IFN pathway had been studied in relation to PD, such as STING linked to mitochondrial dysfunction [56], it is the first time that dysfunctional type I IFN signaling pathway as whole is found associated with SPD.

Among the confirmed genes in the type I IFN signaling pathway, we identified PIAS2, the specific negative regulator of the IFN $\beta$-IFNAR signaling [22], i.e., via specific inhibition of STAT2 [21] to impact the survival and function of neurons. Moreover, PIAS2 was significantly raised in neurons in the brains of SPD, and in particular in SPDD patients, paralleling our finding in Ifn $b^{-/-}$ mice. By knocking down PIAS2 in the brain of Ifn $b^{-1-}$ mice, the PD pathology and clinical manifestations were reversed. We next established that high expression of neuronal PIAS2 in vivo was sufficient to cause PDD-like pathology, and behavioral defects including cognitive impairments in healthy mice. PIAS2 overexpression triggered mitophagy block, resulting in damaged mitochondrial accumulation and elevated oxidative stress including increased oxidative DJ1, and in turn it inhibited ERK1/2 and P53 signaling by reducing their phosphorylation (Supplementary Fig. S4).

Our current findings are significant particularly because the molecular understanding of PD has been based primarily on the discovery of rare familial gene mutations, and although sporadic disease constitutes up to $95 \%$ of all PD cases $[5,6]$ and has been associated with such genes, its etiology remains unknown. Degenerative PD is generally regarded as a movement disorder, but up to $80 \%$ of patients will also develop dementia [57]. The importance of functional dopamine and dopamine-producing neurons in relation to movement disorder has received significant attention in the disease progression, but the underlying molecular mechanisms that drive the advancement from the loss of DA neurons to dementia are poorly understood.

Recent advances in genotyping by GWASs have enabled genetic risk factors that are associated with familial PD to be identified, but no evident gene or molecular factor has surfaced in the onset and progression of idiopathic disease. Furthermore, if overly stringent criteria are applied in GWASs, significant genes with an otherwise weak association with the pathology could be inadvertently disregarded, particularly in sporadic PD. Thus, if 

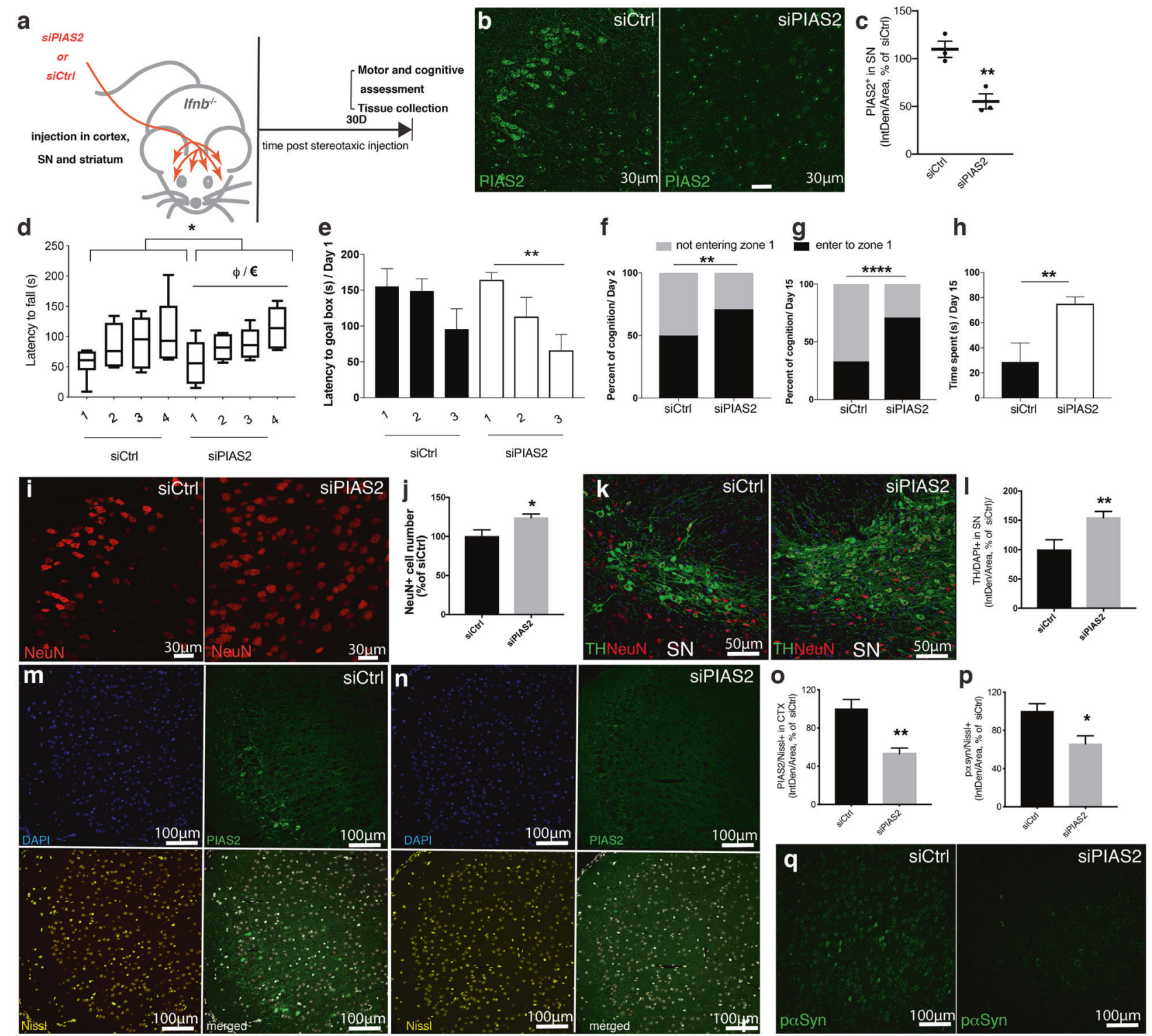

Fig. 5 Brain-specific knockdown of PIAS2 reverses the neurodegenerative process and prevents motor and cognitive impairments in Ifn $^{-/-}$mice. a Schematic of experiment showing injection sites of siRNA knockdown of PIAS2 (siPIAS2) or control (siCtrl) by in vivo stereotactic injection in the frontal cortex and nigrostriatal regions in Ifn $b^{-1-}$ brains and behavioral testing days. b, c Assessment of knockdown efficiency by IF. b IF of PIAS2 (green) in the SN and c quantification in Nissl ${ }^{+}$neurons in the SN. ${ }^{* *} P<0.01$ by two-tailed unpaired Student's $t$-test. $n=3$ / group. $\mathbf{d}$ Rotarod motor coordination test \pm SEM; ${ }^{*} P<0.05$ by two-way ANOVA comparing siCtrl- and siPIAS2-treated groups $(n=6-7)$ and ${ }^{\epsilon} P$ $<0.05$ by repeated measure one-way ANOVA in siPIAS2-treated Ifn $b^{-/-}$mice. One-way ANOVA showing ${ }^{\varphi} P<0.05$ by paired $t$-test between the 1 st and 4th trials in siPIAS2-treated Ifn ${ }^{-/-}$mice. e-h Barnes maze test. e Data latency to goal box in seconds \pm SEM. ${ }^{* *} P<0.01$ by two-tailed paired $t$-test in siPIAS2-treated group indicating improvement from the 1 st to 3rd trial $(n=6-7)$. f, $\mathbf{g}$ Data are percentage of cognition (entering zone 1 or not) during probe days 2 and 15 post training day $1 .{ }^{* *} P<0.01$ and ${ }^{* * * *} P<0.0001$ by two-tailed unpaired $t$-test between siPIAS2- and siCtrl-treated Ifnb ${ }^{-/-}$groups $(n=6-7)$. h Cumulative time spent in zone 1. Data \pm SEM; ${ }^{*} P<0.05$ by two-tailed Mann-Whitney test between siPIAS2- and siCtrl-treated Ifn $b^{-\gamma}$ groups $(n=6-7)$. i-o IF of PIAS2 (green) alone or co-stained with Nissl, NeuN, and DAPI (blue) and TH co-stained with NeuN in siPIAS2- vs. siCtrl-treated groups. $\mathbf{i}$ IF of NeuN and $\mathbf{j}$ quantification of NeuN ${ }^{+}$neurons in the CTX. Graph represents intensity of positive staining/area of siPIAS2 normalized to siCtrl. ${ }^{*} P<0.05$ using two-tailed unpaired Student's $t$-test. $n=10 / g r o u p$. $\mathbf{k}$ IF of TH (green), NeuN (red), and DAPI staining in SN. I IF quantification of $\mathrm{TH}^{+} \mathrm{DAPI}^{+}$neurons. Graph represents intensity of double-positive cells in siPIAS2 normalized to siCtrl ${ }^{*} P<0.01$ by unpaired Student's $t$-test; $n=8-9 /$ group. $\mathbf{m}, \mathbf{n}$ IF of PIAS2 (green) and Nissl (yellow) in CTX of siPIAS2 brains normalized to siCtrl group. o Quantification of PIAS2 in CTX. ${ }^{*} P<0.01$ by two-tailed unpaired Student's $t$-test. $n=4 / g$ roup. $\mathbf{p}, \mathbf{q}$ IF of p $\alpha$ syn (green) in $\mathrm{SN}$ and quantification in Nissl+ neurons. ${ }^{*} P<0.05$ by two-tailed unpaired Student's $t$-test. $n=3 /$ group.

several such genes cooperate in a signaling pathway and collectively induce the PD phenotype, they would not be detected.

According to the IPDGC, when loci with weak association are included in the analysis of a GWAS, the estimated heritability of PD increases from approximately 4-27\% [41]. This finding suggests that much of its genetic association lies below the thresholds for significance in the primary analysis, causing many true polygenic risk alleles to be overlooked [58]. Furthermore, PD is precipitated by a minor change in a-syn expression in patients with familial a-syn gene duplication [59] and perhaps by environmental factors that cause the complete dysfunction of otherwise subtle gene defects that coordinate in a given signaling pathway. These data indicate that SPD is not 

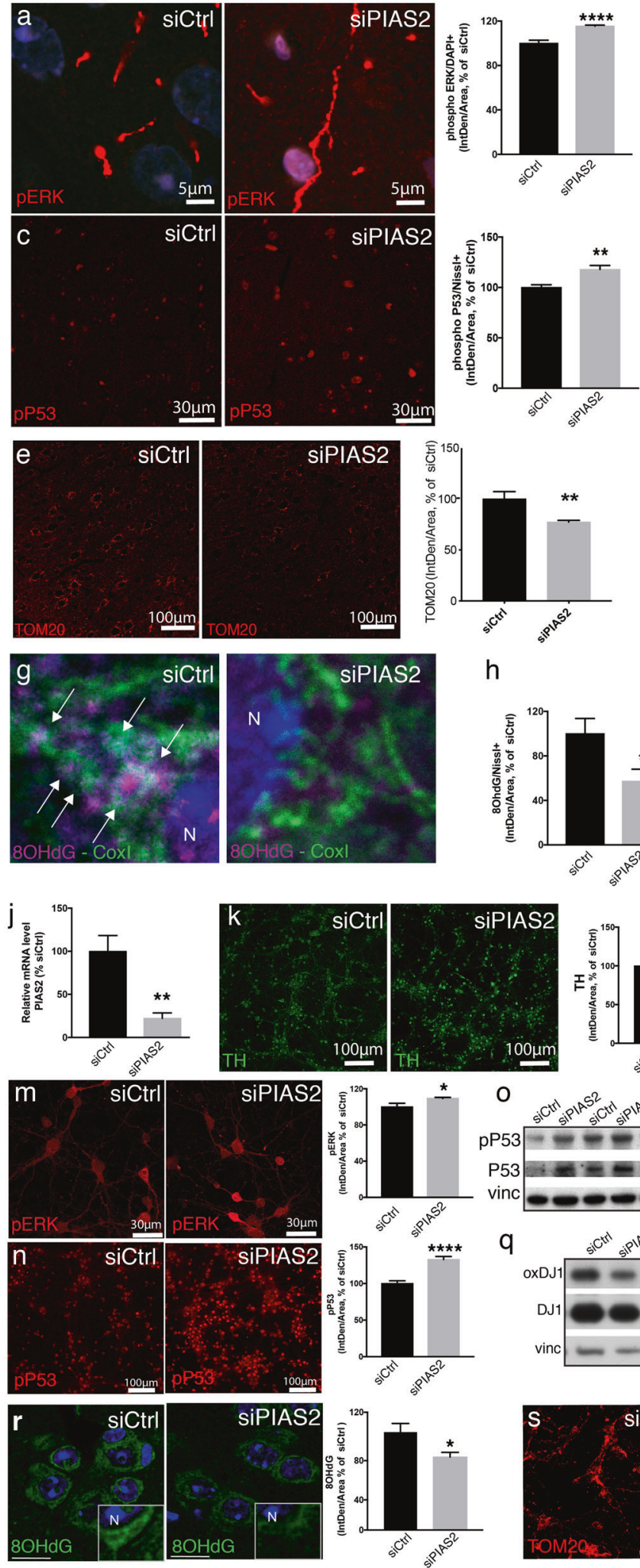

$\mathrm{h}$
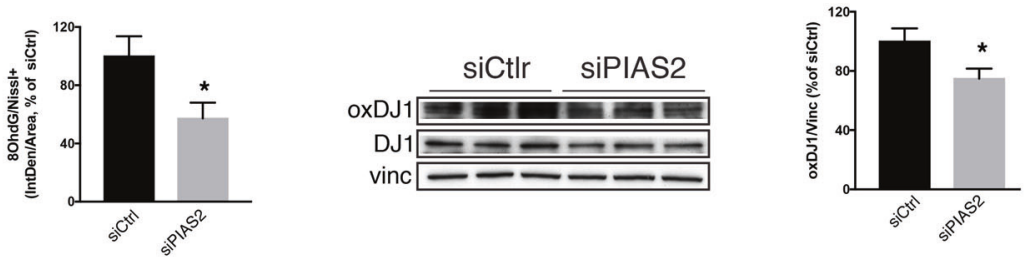

b
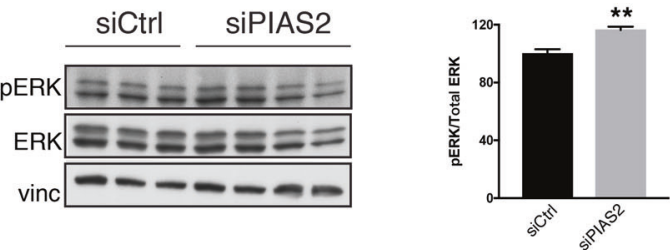

d
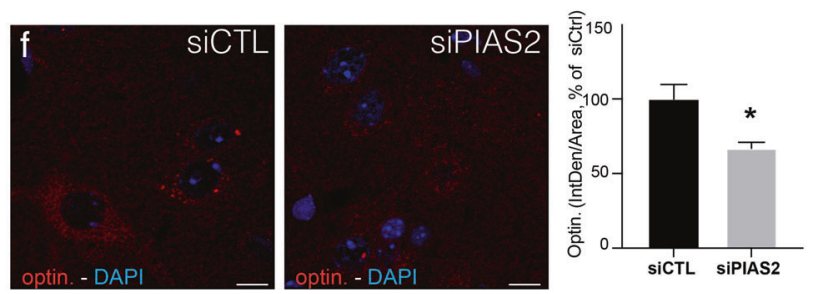

i
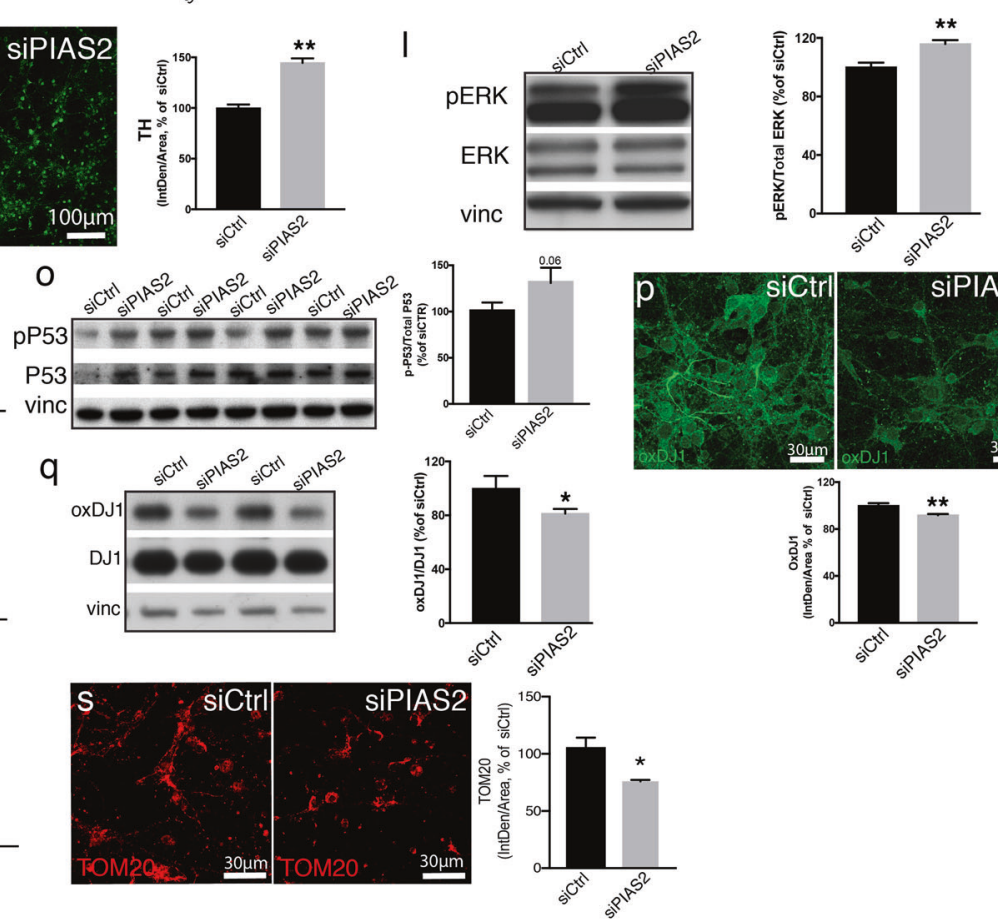

In this study, we tested the hypothesis that SPD arises from the dysregulation of one or more genes, individually or cooperatively, in a specific signaling pathway which determines SPD and its progression to dementia in SPDD. Upon identifying dysregulated type I IFN signaling pathway in SPDD, to further characterize 
Fig. 6 siPIAS2 reverses Ifnb $^{-/-}$neuronal defects by reducing oxidation of DJ1 and improving neuronal pERK- and pP53-mediated mitochondrial and neuronal homeostasis. a-i siRNA knockdown of PIAS2 (siPIAS2) or control (siCtrl) by in vivo stereotactic injection in the frontal cortex and nigrostriatal regions in Ifn $b^{-/-}$brains. a IF staining for phosphorylated (p)ERK1/2 (red) and DAPI (blue) in prefrontal cortex of Ifnb $\mathrm{f}^{-/}$PDD mouse model brain after knockdown of PIAS2 or control. Graph represents mean fluorescence intensity in DAPI-positive cells, normalized to scrambled control; ${ }^{* * *} P<0.0001$ by Student's $t$-test. $n=9 /$ group. $\mathbf{b}$ WB for pERK $1 / 2$ and total ERK1/2 in Ifn $b^{-/-}$mouse brains after knockdown of PIAS2 or control. Vinculin was used as a loading control and values were normalized to scrambled control \pm SEM; ${ }^{* *} P<$ 0.01 by Student's $t$-test. $n=3-4 /$ group. c IF staining for pP53 in prefrontal cortex of Ifnb ${ }^{-/-}$mouse brain after knockdown of PIAS2 or scrambled control. Graphs represent mean fluorescence in $\mathrm{pP}^{+} 3^{+} \mathrm{Nissl}^{+}$neurons normalized to scrambled control; ${ }^{* *} P<0.01$ by Student's $t$-test. $n=7-9 /$ group. d WB for PP53 and total P53 in Ifn ${ }^{-/-}$mouse brain after knockdown of PIAS2 or control. Vinculin was used as a loading control and values were normalized to scrambled control \pm SEM; ${ }^{*} P<0.05$ by Student's $t$-test. $n=3-4 / g r o u p$. e IF staining for TOM20 (red) and quantification of int.dens. in SN. ${ }^{* *} P<0.01$ by two-tailed unpaired Student's $t$-test. $n=5 /$ group. $f$ IF staining for Optin. (red) in striatum and quantification. Scale bars equal 10 microns. Graph represents intensity of 30 neurons in siPIAS2 normalized to siCtrl. ${ }^{*} P<0.01$ by two-tailed unpaired Student's t-test. $n=3$ /group. g IF staining of Coxl and 8OHdG. White arrows indicate colocalization region. Scale bars equal 1 micron. h Quantification of IF staining for $8 \mathrm{OHdG}$ in striatum and prefrontal cortex of $\mathrm{Ifn}^{-/-}$mouse brain after knockdown of PIAS2 or scrambled control. Graph represents mean fluorescence intensity of $8 \mathrm{OHdG}$ in Niss ${ }^{+}$neurons, normalized to scrambled control; ${ }^{*} P<0.05$ by Student's $t$ test. $n=7 /$ group. Images are shown in Supplementary Fig. S3. i WB for oxidized DJ1 and total DJ1 in Ifnh ${ }^{-/-}$mouse brain after knockdown of PIAS2 or scrambled control. Vinculin was used as a loading control and normalized to scrambled contro $I \pm$ SEM; ${ }^{*} P<0.05$ by Student's $t$-test. $n$ =6/group. j-s siRNA knockdown of PIAS2 (siPIAS2) or control (siCtrl) primary CN j RT-qPCR showing fold-change in PIAS2. Data are normalized to scrambled control \pm SEM; ${ }^{* *} P<0.01$ by Student's $t$-test. $n=3$ /group. $\mathbf{j}$ IF staining of TH. Graph represents mean fluorescence intensity and are normalized to scrambled control \pm SEM; ${ }^{* *} P<0.01$ by Student's $t$-test. $n=3 /$ group. $\mathbf{k}$ Representative WB for pERK1/2 and total ERK1/2. Vinculin was used as a loading control, and data are normalized to scrambled control \pm SEM; ${ }^{*} P<0.01$ by Student's $t$-test. $n=3-4 / g r o u p$. I IF staining for pERK1/2. Graph represents mean fluorescence intensity in $p E R K 1 / 2^{+} \mathrm{DAPI}^{+}$cells, normalized to scrambled control; ${ }^{*} P<0.05$ by Student's $t$-test. $n=3-5 /$ group. m IF staining for pP53. Graphs represent mean fluorescence in pP53 $3^{+}$cells, presented as $\%$ of control; ${ }^{* * * * P<}$ 0.0001 by Student's $t$-test. $n=3$ /group. $\mathbf{n}$ WB for pP53 and total P53. Vinculin was used as a loading control, and data are normalized to scrambled control \pm SEM. $P=0.06$ by Student's $t$-test. $n=4$ /group. $o$ Representative WB for phosphorylated and pan P53. Data are normalized to scrambled control \pm SEM. ${ }^{*} P<0.05$ by Student's $t$-test. $\mathbf{p}$ IF staining for oxidized DJ1. Graph represents mean fluorescence intensity of oxDJ1 in Nissl ${ }^{+}$neurons, presented as \% of control; ${ }^{*} P<0.01$ by Student's $t$-test. q Representative WB for oxidized and total DJ1. Data are normalized to scrambled control \pm SEM. ${ }^{*} \mathrm{P}<0.05$ by Student's t-test. $\mathbf{r}$ IF staining for $80 \mathrm{OHG}$. Graph represents mean fluorescence of $80 H d G$ in $\mathrm{Niss}^{+}$neurons, normalized to scrambled control. Close up shows non-nuclear localized $80 \mathrm{OHG}$ positive staining. $\mathrm{N}$ indicates nuclei. ${ }^{*} P<0.05$ by Student's $t$-test. $n=7 /$ group. $\mathbf{s}$ IF staining for TOM20. Graph represents mean fluorescence intensity normalized to scrambled control; ${ }^{* *} P<0.01$ by Student's $t$-test. $n=5 /$ group.

candidate disease-associated genes that had sequence variants in this pathway, we performed a meta-analysis of earlier GWASs on PD patients and detected many factors in IFNAR signaling that were enriched versus HCs. Thus, although these variants did not pass the stringent significance thresholds in the original GWASs, our results imply that this category of genes is pertinent to sPD.

Through transcriptomic analysis, we identified PIAS2 upregulation in all SPD patients with further increase in SPDD patients and established its pathogenic role. There are little data on the involvement of PIAS2 in PD pathology, and its contribution to PD and dementia has not been established. The JAK-STAT pathway, of which PIAS2 is a downstream effector [21], has been suggested to be associated with neurogenesis and to elicit neuroprotective signaling, but its blockade reduces microglia-mediated neuroinflammation in a model of PD $[60,61]$. Furthermore, PIAS2 drives postsynaptic dendritic morphogenesis [62], albeit pertaining more to brain development and plasticity. These findings have created ambiguity with regard to the actual function of this pathway in PD, necessitating the identification of the molecular defects and pertinent cell types in this disease.

Consequently, we determined the contribution of PIAS2 to PDD. Neuronal expression of PIAS2 in the SN, STR, and prefrontal cortex of WT mice caused significant behavioral defects, including movement disorders, cognitive decline, and the loss of dopaminergic neurons-main hallmarks of PDD. Furthermore, we observed that PIAS2 has a negative impact on neuronal action potentials, which is compatible with its potential role in driving the cognitive decline in SPDD.

Based on these findings, our newly identified dysfunctional type 1 IFN signaling pathway, via PIAS2, is the first revelation of the involvement of an entire signaling cascade in the evolution of PD, including its development to dementia. Also, PIAS2 has been reported to interact functionally or physically with many components in this pathway, albeit in contexts other than PD. Based on our data, PIAS2 has potentially compartment-specific functions in neurons, regulating IFN $\beta$-IFNAR signaling in the nucleus and cytoplasm with pERK1/2 and pP53 [63] as partners.
For example, PIAS2 could negatively regulate type 1 IFN target genes by blocking STAT2 while governing the transcriptional expression of such genes as PARK7 [64], UCP2 [65], and TH, which are important for the regulation of neuronal oxidative phosphorylation and DA neuron survival. This differential regulation is consistent with the activity of PIAS2 in upregulating or downregulating target genes in MAPK signaling, depending on whether it is activated by ERK or p38 signaling [66]. A role for pERK/pP53 is also reported in autophagy regulation [52, 53]. In support, we observed that neuronal PIAS2OE upon blocking phosphorylation of ERK/P53 was associated with auto/mitophagy block and hence accumulation of damaged mitochondria. Previously, we have shown that neuronal lack of IFN $\beta$-IFNAR signaling blocks auto/ mitophagy and results in lack of cellular a-syn clearance, and hence its pathological accumulation [9, 32, 67, 68], In support, here we confirmed that PIAS2OE contributes to the accumulation of pathological a-syn and dysfunctional mitochondria with raise in oxidative stress markers, like oxDJ1, associated with inducing a mitophagy block, a process linked to neurodegenerative diseases including PD [69, 70].

PIAS2 is reported to regulate DJ1 activity [50], a transcriptional modulator, notably of P53 [71] and ERK [72], and antioxidant that has been linked extensively to PD. DJ1 has been reported independently to be a PD-associated protein, and the DJ1 L166P mutation is a rare genotype of the disease $[73,74]$. The L166P mutation renders DJ1 improperly SUMOylated and prone to aggregation, causing it to colocalize with tau and a-syn in patients with Pick disease and multiple system atrophy, two neurodegenerative diseases $[75,76]$. In our study, PIAS2OE in neurons upregulated $D J 1$ mRNA while increasing primarily its oxidation; accordingly, oxDJ1 levels are elevated in PD as a result of oxidative stress [77]. DJ1 governed by PIAS2 might act as an antioxidant by activating signaling factors, such as $\mathrm{p} 53$, to restore the redox balance [71]. Independently of DJ1, ERK signaling has been suggested to control p53 activity and thus regulate DNA damageinduced neuronal death [78]. Although our data presented here strongly support a major role for PIAS2 by blocking the classical 
IFN $\beta$-IFNAR signaling pathway, i.e., counteracting JAK-STAT2, $\mathrm{pERK} / \mathrm{pP} 53$, its downstream mitophagy and upregulating oxidative stress including oxidation of DJ1, a role for PIAS2 through SUMOylation regulation could not be excluded, particularly given the pleiotropism of the type I IFN pathway.

Thus, our findings imply that the dysregulation of PIAS2 potentiates the development of PD on several levels against an array of signaling proteins, the multifaceted functions of which converge to disrupt neuronal homeostasis and functions. Collectively, our novel findings render PIAS2 an exciting target for future therapeutic approaches in PD.

\section{REFERENCES}

1. Braak H, Del Tredici K, Rub U, de Vos RA, Jansen Steur EN, Braak E. Staging of brain pathology related to sporadic Parkinson's disease. Neurobiol Aging. 2003:24:197-211.

2. Garcia-Ptacek S, Kramberger MG. Parkinson disease and dementia. J Geriatr Psychiatry Neurol. 2016;29:261-70.

3. Simon-Sanchez J, Schulte C, Bras JM, Sharma M, Gibbs JR, Berg D, et al. Genomewide association study reveals genetic risk underlying Parkinson's disease. Nat Genet. 2009;41:1308-12.

4. International Parkinson Disease Genomics Consortium, Nalls MA, Plagnol V, Hernandez DG, Sharma M, Sheerin UM, et al. Imputation of sequence variants for identification of genetic risks for Parkinson's disease: a meta-analysis of genomewide association studies. Lancet. 2011;377:641-9.

5. Klein C, Westenberger A. Genetics of Parkinson's disease. Cold Spring Harb Perspect Med. 2012;2:a008888.

6. Thomas B, Beal MF. Parkinson's disease. Hum Mol Genet. 2007;16:R183-94. Spec No. 2

7. Evangelou E, loannidis JP. Meta-analysis methods for genome-wide association studies and beyond. Nat Rev Genet. 2013;14:379-89.

8. Subramanian A, Tamayo P, Mootha VK, Mukherjee S, Ebert BL, Gillette MA, et al. Gene set enrichment analysis: a knowledge-based approach for interpreting genome-wide expression profiles. Proc Natl Acad Sci USA. 2005;102:15545-50.

9. Ejlerskov P, Hultberg JG, Wang J, Carlsson R, Ambjorn M, Kuss M, et al. Lack of neuronal IFN-beta-IFNAR causes Lewy body- and Parkinson's disease-like dementia. Cell. 2015;163:324-39.

10. Teige I, Treschow A, Teige A, Mattsson R, Navikas V, Leanderson T, et al. IFN-beta gene deletion leads to augmented and chronic demyelinating experimental autoimmune encephalomyelitis. J Immunol. 2003;170:4776-84.

11. Matsumoto M, Funami K, Oshiumi H, Seya T. Toll-like receptor 3: a link between toll-like receptor, interferon and viruses. Microbiol Immunol. 2004;48:147-54.

12. Teige I, Liu Y, Issazadeh-Navikas S. IFN-beta inhibits T cell activation capacity of central nervous system APCs. J Immunol. 2006;177:3542-53.

13. Prinz $M$, Schmidt $H$, Mildner A, Knobeloch KP, Hanisch UK, Raasch J, et al. Distinct and nonredundant in vivo functions of IFNAR on myeloid cells limit autoimmunity in the central nervous system. Immunity. 2008:28:675-86.

14. Liu Y, Carlsson R, Comabella M, Wang J, Kosicki M, Carrion B, et al. FoxA1 directs the lineage and immunosuppressive properties of a novel regulatory $T$ cell population in EAE and MS. Nat Med. 2014;20:272-82.

15. Rothhammer V, Mascanfroni ID, Bunse L, Takenaka MC, Kenison JE, Mayo L, et al. Type I interferons and microbial metabolites of tryptophan modulate astrocyte activity and central nervous system inflammation via the aryl hydrocarbon receptor. Nat Med. 2016;22:586-97.

16. Lee JK, Tran T, Tansey MG. Neuroinflammation in Parkinson's disease. J Neuroimmune Pharm. 2009;4:419-29.

17. Seth RB, Sun L, Ea CK, Chen ZJ. Identification and characterization of MAVS, a mitochondrial antiviral signaling protein that activates NF-kappaB and IRF 3. Cell. 2005;122:669-82.

18. Yoneyama $M$, Kikuchi $M$, Natsukawa $T$, Shinobu $N$, Imaizumi $T$, Miyagishi $M$, et al. The RNA helicase RIG-I has an essential function in double-stranded RNA-induced innate antiviral responses. Nat Immunol. 2004;5:730-7.

19. Sun L, Wu J, Du F, Chen X, Chen ZJ. Cyclic GMP-AMP synthase is a cytosolic DNA sensor that activates the type I interferon pathway. Science. 2013;339:786-91.

20. Blaszczyk K, Nowicka H, Kostyrko K, Antonczyk A, Wesoly J, Bluyssen HA. The unique role of STAT2 in constitutive and IFN-induced transcription and antiviral responses. Cytokine Growth Factor Rev. 2016;29:71-81.

21. Arora T, Liu B, He H, Kim J, Murphy TL, Murphy KM, et al. PIASx is a transcriptional co-repressor of signal transducer and activator of transcription 4. J Biol Chem. 2003;278:21327-30.

22. Zu S, Xue Q, He Z, Shi C, Wu W, Zhang J, et al. Duck PIAS2 negatively regulates RIG-I mediated IFN- $\beta$ production by interacting with IRF7. Dev Comp Immunol. 2020;108:103664.
23. Jackson PK. A new RING for SUMO: wrestling transcriptional responses into nuclear bodies with PIAS family E3 SUMO ligases. Genes Dev. 2001;15:3053-8.

24. Rott R, Szargel R, Shani V, Hamza H, Savyon M, Abd Elghani F, et al. SUMOylation and ubiquitination reciprocally regulate alpha-synuclein degradation and pathological aggregation. Proc Natl Acad Sci USA. 2017;114:13176-81.

25. Stamper C, Siegel A, Liang WS, Pearson JV, Stephan DA, Shill H, et al. Neuronal gene expression correlates of Parkinson's disease with dementia. Mov Disord. 2008;23:1588-95.

26. Kutmon M, Riutta A, Nunes N, Hanspers K, Willighagen EL, Bohler A, et al. WikiPathways: capturing the full diversity of pathway knowledge. Nucleic Acids Res. 2016;44:D488-94.

27. Kutmon M, van lersel MP, Bohler A, Kelder T, Nunes N, Pico AR, et al. PathVisio 3: an extendable pathway analysis toolbox. PLoS computational Biol. 2015;11: e1004085.

28. Nalls MA, Pankratz N, Lill CM, Do CB, Hernandez DG, Saad M, et al. Large-scale meta-analysis of genome-wide association data identifies six new risk loci for Parkinson's disease. Nat Genet. 2014;46:989-93.

29. Grimm D, Kay MA, Kleinschmidt JA. Helper virus-free, optically controllable, and two-plasmid-based production of adeno-associated virus vectors of serotypes 1 to 6. Mol Ther. 2003;7:839-50.

30. Liu Y, Teige I, Birnir B, Issazadeh-Navikas S. Neuron-mediated generation of regulatory $T$ cells from encephalitogenic $T$ cells suppresses EAE. Nat Med. 2006;12:518-25.

31. Brewer GJ, Torricelli JR. Isolation and culture of adult neurons and neurospheres. Nat Protoc. 2007;2:1490-8.

32. Ambjorn M, Ejlerskov $P$, Liu $Y$, Lees $M$, Jaattela $M$, Issazadeh-Navikas S. IFNB1/ interferon-beta-induced autophagy in MCF-7 breast cancer cells counteracts its proapoptotic function. Autophagy. 2013;9:287-302.

33. Ejlerskov $P$, Rasmussen I, Nielsen $T$, Bergstrom AL, Tohyama $Y$, Jensen $P H$, et al. Tubulin polymerization-promoting protein (TPPP/p25alpha) promotes unconventional secretion of alpha-synuclein through exophagy by impairing autophagosome-lysosome fusion. J Biol Chem. 2013;288:17313-35.

34. Brown NA, Kemp JA, Seabrook GR. Block of human voltage-sensitive $\mathrm{Na}+$ currents in differentiated SH-SY5Y cells by lifarizine. Br J Pharm. 1994;113:600-6.

35. Lesnick TG, Papapetropoulos S, Mash DC, Ffrench-Mullen J, Shehadeh L, de Andrade $\mathrm{M}$, et al. A genomic pathway approach to a complex disease: axon guidance and Parkinson disease. PLoS Genet. 2007;3:e98.

36. Zheng B, Liao Z, Locascio JJ, Lesniak KA, Roderick SS, Watt ML, et al. PGC-1alpha, a potential therapeutic target for early intervention in Parkinson's disease. Sci Transl Med. 2010;2:52ra73.

37. Dijkstra AA, Ingrassia A, de Menezes RX, van Kesteren RE, Rozemuller AJ, Heutink $P$, et al. Evidence for immune response, axonal dysfunction and reduced endocytosis in the substantia nigra in early stage Parkinson's disease. PLoS One. 2015;10:e0128651.

38. Honda K, Taniguchi T. IRFs: master regulators of signalling by Toll-like receptors and cytosolic pattern-recognition receptors. Nat Rev Immunol. 2006;6:644-58.

39. Liu DJ, Peloso GM, Zhan X, Holmen OL, Zawistowski M, Feng S, et al. Metaanalysis of gene-level tests for rare variant association. Nat Genet. 2014;46:200-4.

40. Jansen IE, Bras JM, Lesage S, Schulte C, Gibbs JR, Nalls MA, et al. CHCHD2 and Parkinson's disease. Lancet Neurol. 2015;14:678-9.

41. Holmans $P$, Moskvina V, Jones $L$, Sharma $M$, International Parkinson's Disease Genomics Consortium, Vedernikov A, et al. A pathway-based analysis provides additional support for an immune-related genetic susceptibility to Parkinson's disease. Hum Mol Genet. 2013;22:1039-49.

42. Nalls MA, Blauwendraat $C$, Vallerga $C L$, Heilbron $K$, Bandres-Ciga $S$, Chang $D$, et al. Identification of novel risk loci, causal insights, and heritable risk for Parkinson's disease: a meta-analysis of genome-wide association studies. Lancet Neurol. 2019;18:1091-102.

43. Cassarino DS, Fall CP, Swerdlow RH, Smith TS, Halvorsen EM, Miller SW, et al. Elevated reactive oxygen species and antioxidant enzyme activities in animal and cellular models of Parkinson's disease. Biochim Biophys Acta. 1997;1362:77-86.

44. Hadjilambreva G, Mix E, Rolfs A, Muller J, Strauss U. Neuromodulation by a cytokine: interferon-beta differentially augments neocortical neuronal activity and excitability. J Neurophysiol. 2005;93:843-52.

45. Nishida T, Yasuda H. PIAS1 and PIASxalpha function as SUMO-E3 ligases toward androgen receptor and repress androgen receptor-dependent transcription. J Biol Chem. 2002;277:41311-7.

46. Grunewald A, Kumar KR, Sue CM. New insights into the complex role of mitochondria in Parkinson's disease. Prog Neurobiol. 2019;177:73-93.

47. Liu XL, Wang YD, Yu XM, Li DW, Li GR. Mitochondria-mediated damage to dopaminergic neurons in Parkinson's disease (Review). Int J Mol Med. 2018;41:615-23. 
48. Bonifati V, Rizzu P, Squitieri F, Krieger E, Vanacore N, van Swieten JC, et al. DJ-1 (PARK7), a novel gene for autosomal recessive, early onset parkinsonism. Neurol Sci. 2003;24:159-60.

49. Takahashi-Niki K, Niki T, Iguchi-Ariga S, Ariga H. [Function of DJ-1 in mitochondria]. Yakugaku Zasshi. 2012;132:1105-10.

50. Shinbo Y, Niki T, Taira T, Ooe H, Takahashi-Niki K, Maita C, et al. Proper SUMO-1 conjugation is essential to DJ-1 to exert its full activities. Cell Death Differ. 2006;13:96-108.

51. Kumari R, Sen N, Das S. Tumour suppressor p53: understanding the molecular mechanisms inherent to cancer. Curr Sci. 2014;107:786-94.

52. Brantley-Finley C, Lyle CS, Du L, Goodwin ME, Hall T, Szwedo D, et al. The JNK, ERK and p53 pathways play distinct roles in apoptosis mediated by the antitumor agents vinblastine, doxorubicin, and etoposide. Biochem Pharm. 2003;66:459-69.

53. Zhang X, Cheng Q, Yin H, Yang G. Regulation of autophagy and EMT by the interplay between p53 and RAS during cancer progression (Review). Int J Oncol. 2017;51:18-24.

54. Jiang $T$, Sun $Q$, Chen S. Oxidative stress: a major pathogenesis and potential therapeutic target of antioxidative agents in Parkinson's disease and Alzheimer's disease. Prog Neurobiol. 2016;147:1-19.

55. lacovitti L, Lee J, Joh TH, Reis DJ. Expression of tyrosine hydroxylase in neurons of cultured cerebral cortex: evidence for phenotypic plasticity in neurons of the CNS. J Neurosci. 1987;7:1264-70.

56. Sliter DA, Martinez J, Hao L, Chen X, Sun N, Fischer TD, et al. Parkin and PINK1 mitigate STING-induced inflammation. Nature. 2018;561:258-62.

57. Aarsland D, Kurz MW. The epidemiology of dementia associated with Parkinson disease. J neurological Sci. 2010;289:18-22.

58. Keller MF, Saad M, Bras J, Bettella F, Nicolaou N, Simon-Sanchez J, et al. Using genome-wide complex trait analysis to quantify 'missing heritability' in Parkinson's disease. Hum Mol Genet. 2012;21:4996-5009.

59. Kim MJ, Woo JS, Kwon CH, Kim JH, Kim YK, Kim KH. Luteolin induces apoptotic cell death through AIF nuclear translocation mediated by activation of ERK and p38 in human breast cancer cell lines. Cell Biol Int. 2012;36:339-44.

60. Wang T, Yuan W, Liu Y, Zhang Y, Wang Z, Zhou X, et al. The role of the JAK-STAT pathway in neural stem cells, neural progenitor cells and reactive astrocytes after spinal cord injury. Biomed Rep. 2015;3:141-6.

61. Qin H, Buckley JA, Li X, Liu Y, Fox TH III, Meares GP, et al. Inhibition of the JAK/ STAT pathway protects against alpha-synuclein-induced neuroinflammation and dopaminergic neurodegeneration. J Neurosci. 2016;36:5144-59.

62. Shalizi A, Bilimoria PM, Stegmuller J, Gaudilliere B, Yang Y, Shuai K, et al. PIASx is a MEF2 SUMO E3 ligase that promotes postsynaptic dendritic morphogenesis. J Neurosci. 2007;27:10037-46.

63. Schmidt D, Muller S. Members of the PIAS family act as SUMO ligases for c-Jun and p53 and repress p53 activity. Proc Natl Acad Sci USA. 2002;99:2872-7.

64. Oh SE, Park H-J, He L, Skibiel C, Junn E, Mouradian MM. The Parkinson's disease gene product DJ-1 modulates miR-221 to promote neuronal survival against oxidative stress. Redox Biol. 2018;19:62-73.

65. Hwang R-D, Wiemerslage L, LaBreck CJ, Khan M, Kannan K, Wang X, et al. The neuroprotective effect of human uncoupling protein 2 (hUCP2) requires CAMPdependent protein kinase in a toxin model of Parkinson's disease. Neurobiol Dis. 2014;69:180-91.

66. Yang S-H, Sharrocks AD. PIASxalpha differentially regulates the amplitudes of transcriptional responses following activation of the ERK and p38 MAPK pathways. Mol Cell. 2006;22:477-87.

67. Tresse E, Riera-Ponsati L, Jaberi E, Sew WQG, Ruscher K, Issazadeh-Navikas S. IFN-beta rescues neurodegeneration by regulating mitochondrial fission via STAT5, PGAM5, and Drp1. EMBO J. 2021;40:e106868. https://doi.org/10.15252/embj.2020106868.

68. Nehammer C, Ejlerskov P, Gopal S, Handley A, Ng L, Moreira P, et al. Interferonbeta-induced miR-1 alleviates toxic protein accumulation by controlling autophagy. Elife. 2019;8:e49930.

69. Lou G, Palikaras K, Lautrup S, Scheibye-Knudsen M, Tavernarakis N, Fang EF. Mitophagy and neuroprotection. Trends Mol Med. 2020;26:8-20.

70. Pickrell AM, Youle RJ. The roles of PINK1, parkin, and mitochondrial fidelity in Parkinson's disease. Neuron 2015;85:257-73.

71. Dolgacheva LP, Berezhnov AV, Fedotova El, Zinchenko VP, Abramov AY. Role of DJ-1 in the mechanism of pathogenesis of Parkinson's disease. J Bioenerg Biomembr. 2019;51:175-88.

72. Oh SE, Mouradian MM. Cytoprotective mechanisms of DJ-1 against oxidative stress through modulating ERK1/2 and ASK1 signal transduction. Redox Biol. 2018;14:211-7.

73. DJ Moore, Zhang L, Dawson TM, et al. A missense mutation (L166P) in DJ-1, linked to familial Parkinson's disease, confers reduced protein stability and impairs homo-oligomerization. J Neurochem. 2003;87:1558-67.
74. Moore DJ, Zhang L, Troncoso J, Lee MK, Hattori N, Mizuno Y, et al. Association of DJ-1 and parkin mediated by pathogenic DJ-1 mutations and oxidative stress. Hum Mol Genet. 2005;14:71-84.

75. Neumann M, Müller V, Görner K, Kretzschmar HA, Haass C, Kahle PJ. Pathological properties of the Parkinson's disease-associated protein DJ-1 in alphasynucleinopathies and tauopathies: relevance for multiple system atrophy and Pick's disease. Acta Neuropathologica. 2004;107:489-96.

76. Rizzu P, Hinkle DA, Zhukareva V, Bonifati V, Severijnen L-A, Martinez D, et al. DJ-1 colocalizes with tau inclusions: a link between parkinsonism and dementia. Ann Neurol. 2004;55:113-8.

77. Mita Y, Kataoka Y, Saito Y, Kashi T, Hayashi K, Iwasaki A, et al. Distribution of oxidized DJ-1 in Parkinson's disease-related sites in the brain and in the peripheral tissues: effects of aging and a neurotoxin. Sci Rep. 2018;8:12056-.

78. Lee JH, Kim KT. Regulation of cyclin-dependent kinase 5 and p53 by ERK1/2 pathway in the DNA damage-induced neuronal death. J Cell Physiol. 2007;210:784-97.

\section{ACKNOWLEDGEMENTS}

We would like to thank Natasha Fauerby for help with the in vivo and behavioral experiments. This project received funding from the Lundbeck Foundation (LF ID\#R291-2016-536, R199-2015-2368, and R210-2015-3372), Danish Council for Independent Research-Medicine (DFF-6110-00658, and 6110-00461B), and Alzheimer forskningsfonden. $\mathrm{EH}$ received funding from the Chinese Scholarship Council to facilitate her exchange PhD studies at BRIC, University of Copenhagen.

\section{AUTHOR CONTRIBUTIONS}

$J M, E T, P E, E H, Y L, A M, E M M C$, and RR performed the experiments and analyzed and prepared the data. LS, CFR, AS-Z, and JBA performed the bioinformatics and GSEA analysis. SA, TB, and TG supplied the PD patient materials. MS and TG performed the meta-analysis. KK assisted with the AAV virus selection and production. MP provided the Nes-Cre Ifnar $1^{\mathrm{fl} / \mathrm{fl}}$ mice. SI-N designed and supervised the study, analyzed and interpreted the data, and wrote the initial manuscript. All authors read and contributed to the final manuscript.

\section{COMPETING INTERESTS}

The authors declare no competing interests.

\section{ADDITIONAL INFORMATION}

Supplementary information The online version contains supplementary material available at https://doi.org/10.1038/s41380-021-01207-w.

Correspondence and requests for materials should be addressed to S.I.-N.

Reprints and permission information is available at http://www.nature.com/ reprints

Publisher's note Springer Nature remains neutral with regard to jurisdictional claims in published maps and institutional affiliations.

Open Access This article is licensed under a Creative Commons Attribution 4.0 International License, which permits use, sharing, adaptation, distribution and reproduction in any medium or format, as long as you give appropriate credit to the original author(s) and the source, provide a link to the Creative Commons license, and indicate if changes were made. The images or other third party material in this article are included in the article's Creative Commons license, unless indicated otherwise in a credit line to the material. If material is not included in the article's Creative Commons license and your intended use is not permitted by statutory regulation or exceeds the permitted use, you will need to obtain permission directly from the copyright holder. To view a copy of this license, visit http://creativecommons. org/licenses/by/4.0/.

(c) The Author(s) 2021 